\title{
Analysis of polymorphisms in the circadian- related genes and breast cancer risk in Norwegian nurses working night shifts
}

Shanbeh Zienolddiny ${ }^{1 *}$, Aage Haugen ${ }^{1}$, Jenny-Anne Sigstad Lie ${ }^{2}$, Helge Kjuus ${ }^{2}$, Kristine Haugen Anmarkrud ${ }^{1}$ and Kristina Kjærheim ${ }^{3}$

\begin{abstract}
Introduction: Some studies have suggested that night work may be associated with an increased risk of breast cancer in nurses. We aimed to explore the role of circadian gene polymorphisms in the susceptibility to night work-related breast cancer risk.

Methods: We conducted a nested case-control study of Norwegian nurses comprising 563 breast cancer cases and 619 controls within a cohort of 49,402 Norwegian nurses ages 35 to 74 years. We studied 60 single-nucleotide polymorphisms (SNPs) in 17 genes involved in the regulation of the circadian rhythm in cases and controls. The data were analyzed in relation to the two exposure variables "maximum number of consecutive night shifts ever worked" and "maximum number of consecutive night shifts worked for at least 5 years." The odds of breast cancer associated with each SNP was calculated in the main effects analysis and in relation to night shift work. The statistically significant odds ratios were tested for noteworthiness using two Bayesian tests: false positive report probability (FPRP) and Bayesian false discovery probability (BFDP).

Results: In the main effects analysis, CC carriers of rs4238989 and GG carriers of rs3760138 in the AANAT gene had increased risk of breast cancer, whereas $T$ carriers of BMAL1 rs2278749 and TT carriers of CLOCK rs3749474 had reduced risk. The associations were found to be noteworthy using both the FPRP and BFDP tests. With regard to the effect of polymorphisms and night work, several significant associations were observed. After applying FPRP and BFDP in women with at least four night shifts, an increased risk of breast cancer was associated with variant alleles of SNPs in the genes AANAT (rs3760138, rs4238989), BMAL1 (rs2290035, rs2278749, rs969485) and ROR-b (rs3750420). In women with three consecutive night shifts, a reduced risk of breast cancer was associated with carriage of variant alleles of SNPs in CLOCK (rs3749474), BMAL1 (rs2278749), BMAL2 (rs2306074), CSNK1E (rs5757037), NPAS2 (rs17024926), ROR-b (rs3903529, rs3750420), MTNR1A (rs131113549) and PER3 (rs1012477).
\end{abstract}

Conclusions: Significant and noteworthy associations between several polymorphisms in circadian genes, night work and breast cancer risk were found among nurses who had worked at least three consecutive night shifts.

Keywords: Breast cancer, Circadian, Clock, Night shift, SNP

\section{Introduction}

Breast cancer in women is the second leading cause of mortality [1]. The etiology of breast cancer involves several risk factors, such as early age at menarche, late age at menopause, late age at first birth, nulliparity, hormonal

\footnotetext{
* Correspondence: shan.zienolddiny@stami.no

'Department of Chemical and Biological Work Environment, National

Institute of Occupational Health, PB 8149 Department, N-0033 Oslo, Norway

Full list of author information is available at the end of the article
}

factors, ionizing radiation exposure and alcohol consumption $[2,3]$. However, these known risk factors do not fully explain the high incidence of breast cancer. Genetic factors are also involved in the etiology of breast cancer, including rare high-risk mutations (BRCA1 and $B R C A 2)$, more moderate susceptibility variants $(C H E K 2, A T M)$ and several as yet unidentified common susceptibility variants associated with low to moderate increased risk $[4,5]$.

\section{Biomed Central}


At present, there is not sufficient evidence for any occupational exposure to be classified as carcinogenic in relation to breast cancer. However, night work is a potential risk factor that has been found in many studies to be associated with an elevated risk of breast cancer [6-12]. A recent study reported that $4.6 \%$ of all female breast cancers could be attributed to occupational exposure to shift work [13]. This is important, given the large proportion of women engaged in shift work and the very high incidence of female breast cancer in general. Although the majority of epidemiological studies are suggestive of a positive association, not all studies show a consistent association between night work and increased risk of breast cancer [14-16].

Night work was classified as a Group 2A carcinogen by the International Agency for Research on Cancer (IARC) in 2007 [17,18]. The hypothesis of circadian disruption by light at night (LAN) has received particular attention in this evaluation [19]. Disruption of the circadian rhythm has been hypothesized to increase cancer risk through its impact on the production of the circadian hormone melatonin $[20,21]$. A potential mechanism could be through suppression of the nocturnal production of melatonin, caused by exposure to LAN, which may also influence the patterns of sex hormone production and in turn influence breast cancer risk [22,23]. The levels of melatonin are regulated through its biosynthesis from the amino acid tryptophan, which is mediated by the arylalkylamine $N$-acetyltransferase (AANAT) protein or melatonin receptors MTNR1A (MT1) and MTNR1B (MT2) and possibly the retinoic acid-related orphan receptors ROR-a and ROR-b [24]. An antiproliferative effect of melatonin has also been shown in human breast cancer cell lines. In estrogen receptor-positive $\left(E R^{+}\right)$ human breast cancer cells, melatonin suppresses both ER mRNA expression and estrogen-induced transcriptional activity of the ER $[25,26]$. Melatonin also regulates the transcriptional activity of other enzymes involved in estrogen metabolism and the expression of circadian and circadian-targeted genes. Recently, melatonin was found to induce apoptosis [27] and to reduce the levels of oxidative stress $[28,29]$. Other mechanisms, such as impact of sleep disruption on human physiological processes, may also be involved [30], although not all the results of studies on the impact of sleep disruption on breast cancer risk have been consistent [31,32].

The circadian clock is regulated by several proteins, such as aryl hydrocarbon receptor nuclear translocator-likes (ARNTL or BMAL1, ARNTL2 or BMAL2) also known as Mop3, circadian locomotor output cycle kaput (CLOCK), neuronal PAS domain protein 2 (NPAS2), periods (PER1, PER2 and PER3), cryptochromes (CRY1 and CRY2), TIMELESS, ROR-a and ROR-b, that interact with each other in various transcriptional and translational feedback loops [33]. The main signal is the daily light-dark rotation, which is mediated by light-sensing opsin proteins such as opsin 4 (OPN4) [34].

The circadian genes may affect cancer susceptibility through effects on biological pathways that regulate DNA damage and repair, carcinogen metabolism and/or detoxification, cell growth and cell death [35]. It has been shown that expression of the circadian gene PER 1 may be downregulated in tumors and tumor cell lines compared to normal cells [36]. The PER2 gene has been shown to induce apoptosis through upregulation of the tumor suppressor gene TP53 and the proapoptotic gene Bax [37]. A PER2 gene mutation (S662G) also leads to enhanced resistance to $\mathrm{X}$-ray-induced apoptosis and increased RAS-mediated oncogenic transformation, and it affects tumorigenesis in mice [38].

To date, there are no genetic susceptibility biomarkers identifying women who are particularly susceptible to breast cancer related to exposure to night work. In particular, knowledge of how night work, together with common genetic variants in genes regulating the circadian rhythm, may affect breast cancer risk is limited [39]. The aim of this study was to analyze a number of polymorphisms in the core circadian genes and genes involved in melatonin biosynthesis and melatonin bioavailability as potential biomarkers of breast cancer risk in night workers.

\section{Methods}

\section{Case patients and controls}

The details of study design, data collection, recruitment of cases and controls have been described previously by Lie et al. [8,9]. Briefly (see Additional File 1, Figure S1), a nested case-control study of 1,182 women ages 35 to 74 years was performed within an established national cohort of 49,402 Norwegian nurses who graduated between 1914 and 1985. All of the women had been diagnosed with histologically confirmed invasive breast cancer between 1990 and $2007(n=1,132)$, were alive as of February 2009 ( $n=943)$, consented to be interviewed ( $n=706)$ and had an occupational history as a nurse of at least 1 year $(n=699)$. The controls were frequencymatched to cases by year of diagnosis and in 5-year age groups. They were all cancer-free at and prior to the year of diagnosis of the case, were alive as of February 2009 $(n=1,384)$, had consented to an interview $(n=900)$ and worked as nurses for minimum of 1 year $(n=895)$. Breast cancer cases were identified by linkage to the Cancer Registry of Norway by personal identification number given to all Norwegians alive since 1960. The Cancer Registry of Norway has virtually complete records of all cancer cases in the Norwegian population since January 1953 [40]. Both cases and controls gave their full informed consent that their information could be used 
and published for research purposes, given that their personal details would remain anonymous. The study was approved by the Regional Committee for Medical and Health Research Ethics, South-East region (S-08430a, 2008/10453).

\section{Assessment of night work}

Full details on the exposure assessment were described in a recent study [9]. Briefly, different exposure metrics of night work were computed on the basis of work history. The term night work in this study includes working periods from rotating as well as permanent night schedules. A "night shift" was defined as a shift including work between 12 a.m. and 6 a.m. For each job, we assessed information gathered for the year of start and end, workplace (hospital, other institution and/or others), proportion of full job and work schedule (only day shifts, only night shifts or both day and night shifts). If the job included night shifts, we also asked about the number of night shifts per month, and number of consecutive night shifts. Two surrogate measures for night work were applied: maximum number of consecutive night shifts in all jobs $(<3,3$ and $\geq 4)$ and maximum number of consecutive night shifts the nurse had worked during a minimum of 5 years $(<3,3$ or $\geq 4)$.

We focused our analysis on the variable consecutive night shift because, in our previous analysis [9], the consecutive night shift variable that takes duration into account was the only variable found to be significantly associated with an increased risk of breast cancer. In that previous analysis, a positive trend was seen with an increasing number of consecutive nights, albeit that a significant association was observed only when comparing those with at least 5 years of work in a job including six or more consecutive night shifts with those who never worked nights.

\section{Collection of saliva and DNA extraction}

DNA from 563 saliva samples taken from cases and 619 samples obtained from controls were isolated using the Oragene.DNA isolation kit (DNA Genotek Inc, Kanata, ON, Canada) as instructed by the manufacturer, with some modifications. Briefly, $1 \mathrm{ml}$ of saliva was transferred to an Eppendorf tube, and reagents provided by the supplier were added. DNA was successfully extracted from all saliva samples that were received.

\section{Selection of polymorphisms and genotyping}

The single-nucleotide polymorphism (SNP) selection strategy was based primarily on a candidate gene approach using information from a search of relevant studies published by 2009 . We searched the literature for polymorphisms in the core circadian genes and melatonin biosynthesis and/or bioavailability that had been investigated for an association with cancer risk. Since there were few polymorphisms that had been investigated by 2009 , we also included polymorphisms using one or more of the following criteria: assumed functionality (located in the regulatory regions, for example, 3'-UTR, 5'-UTR or amino acid change), cancer genetic marker of susceptibility (CGEMS) for breast cancer in epidemiological studies (candidate gene or genome-wide association studies (GWASs)), candidate tag SNP with $r^{2} \geq 0.80$ and minor allele frequency (MAF) $\geq$ $5 \%$, and location in the relevant circadian, melatonin and light signaling pathways. Because of the constraint of the project budget, and hence limitations on the number of polymorphisms that could be placed on the chip, 60 SNPs were successfully designed and genotyped (see Additional File 2, Tables S1 and S2). The genotyping success rate was similar in cases and controls: at least 0.80 in cases and 0.82 in controls. All SNPs except for one SNP in the CRY2 gene (rs12364060) and one SNP in the MTNR1A gene (rs2119882) were in Hardy-Weinberg equilibrium. Two previously reported SNPs, rs35670208 in the ARNTL2/ $B M A L 2$ gene and rs28936679 in the AANAT gene, respectively, were found to be nonpolymorphic in this population. Genotyping was performed using the iPLEX genotyping assay (Sequenom Inc, San Diego, CA, USA) at the Centre for Integrative Genetics (CIGENE) genotyping core facility at the University for Environment and Biosciences, Ås, Norway.

\section{Statistical methods}

Pearson's $\chi^{2}$ test was used to assess case-control differences in the frequency distribution of categorical variables, and a nonparametric Mann-Whitney test was used to assess differences in median age between cases and controls. On the basis of existing knowledge of factors associated with breast cancer, the following factors were regarded as potential confounders: age at time of diagnosis (as a continuous variable in 5-years age groups), period of diagnosis (1996 to 1999 and 2000 to 2007), parity (0, 1 or 2 or $\geq 3$ children), family history of breast cancer in mother and/or sister (no or yes), use of hormone treatment during the 2 years before the reference year (no or premenopausal, or yes) and frequency of alcohol consumption at time of diagnosis (less than twice weekly or at least twice per week). These variables were included in the final logistic regression model to obtain adjusted odds ratios (ORs) for developing breast cancer associated with the frequency of polymorphisms alone (main effects analysis), the polymorphisms and night work (stratified by number of consecutive night shifts). On the basis of the results of our previous study showing associations between intensity of night work and breast cancer risk, we examined the simultaneous effects of polymorphisms and night work by comparing the frequency of genotypes of each polymorphism in cases and controls who had worked either at least four 
and three consecutive night shifts with a reference group who had worked only day shifts or a maximum of two consecutive night shifts. We compared the frequency of SNPs in subgroups defined by both intensity (number of consecutive nights) and duration (number of years) of night work. In our previous study [9], the consecutive night shift variable that takes duration into account was the only variable found to be significantly associated with an increased risk of breast cancer, that is, a significant association was observed only when individuals with at least 5 years of work experience in a job including six or more consecutive night shifts were compared with those who had never worked nights. A positive trend was seen with increasing number of consecutive nights, however, thus, to avoid small numbers of subjects in the stratified analyses in the present study, women who had worked at least four consecutive night shifts were classified as the most exposed group. The relative risk of breast cancer associated with each genotype was estimated as OR with 95\% confidence interval (CI) using unconditional logistic regression models. For determination of interactions between the shift work and SNP genotypes, the product of shift work $\times$ genotype variables was added to the model. Interaction $P$ values ( $P$ interaction) are reported for the joint effects of shift work and genotype. All reported $P$ values are two-sided. $P<0.05$ was considered statistically significant. All statistical analyses were carried out using SPSS release 21 software (SPSSInc, Chicago, IL, USA).

\section{Multiple testing and false discovery rates}

To evaluate the possibility of false-positives due to multiple testing, we applied two Bayesian statistical tests, false positive report probability (FPRP) and the Bayesian false discovery probability (BFDP), that have been used frequently to test noteworthiness of findings in genetic association studies [41-43]. The FPRP statistical tool was introduced by Wacholder et al. [42] as a means to assess whether the strength of an association is noteworthy in the case of multiple hypothesis testing. In addition, we used the BFDP, which is a recent further development of FPRP [41]. We performed both FPRP and BFDP tests for each SNP. For both tests, we chose the noteworthiness thresholds (FPRP 0.2 and BFDP 0.8) following the original papers $[41,42]$. We considered noteworthy only those associations that passed both tests. We have set low prior probability values compared to what commonly has been used [44].

\section{Results}

The study design is a nested case-control study (see Additional File 1, Figure S1). The original cohort study of Norwegian nurses was described in detail by Lie et al. $[8,9]$. The demographic characteristics of cases and controls (Table 1) are not significantly different, except for the presence of breast cancer in mother or sister. The exposure parameters and the number of cases and controls in each exposure group are shown in Table 2. The selected circadian genes, chromosome locations and the protein function of each respective gene are shown in Additional File 2, Table S1. The SNPs with corresponding reference SNP (rs) numbers, SNP types, base changes, MAF, Hardy-Weinberg-derived $P$ values and genotyping rates are given in Additional File 2, Table S2.

Sixty SNPs in 17 genes (see Additional File 2, Tables S1 and Table S2) from the circadian signaling pathway were analyzed for association with breast cancer risk. As shown in Additional File 2, Table S2, the MAFs of the SNPs in controls were similar to those reported previously in Caucasian populations, indicating the validity of the genotyping methodology [45-47].

The analysis of main effects, without regard to night work (significant associations are shown in Table 3), showed that four SNPs were associated with breast cancer risk: CC carriers of the AANAT rs4238989 with an OR of 1.43 (95\% CI, 1.01-2.01) and GG carriers of AANAT rs3760138 with an OR of 1.42 (95\% CI, 1.012.01) had increased risk of breast cancer. The carriers of variant TT genotype of SNP rs2278749 in the BMAL1 gene with an OR of 0.45 (95\% CI, 0.23- 0.90) and the homozygote genotype TT of rs3749474 in the CLOCK gene with an OR of 0.64 (95\% CI, 0.45-0.92) had reduced risk of breast cancer. All of these associations were found to be noteworthy for both FPRP $(<0.2)$ and BFDP $(<0.8)$.

Compared to the reference group (Table 4), in the highest exposed group with at least four consecutive night shifts, CLOCK rs11133373 (OR 2.75, 95\% CI 1.07- 7.09), ROR- $b$ rs3903529 (OR 1.87, 95\% CI 1.0- 3.61), two SNPs in the AANAT gene (rs3760138 (OR 1.82, 95\% CI 1.132.93) and rs4238989 (OR 1.62, 95\% CI 1.0- 2.68)) and MTNR1B-rs10830963 (OR 2.48, 95\% CI 1.06- 5.8) were associated with increased risk of breast cancer. In the same group, two SNPs in the CRY2 gene, rs11038689 (OR $0.42,95 \%$ CI $0.17-1.01$ ) and rs1401417 (OR 0.31, 95\% CI $0.10-0.94)$, were associated with reduced risk. In the group with three night shifts, a reduced risk of breast cancer was associated with the presence of one variant allele (heterozygote) or two variant alleles (homozygote) for SNPs in CLOCK rs11133373 (OR 0.50, 95\% CI 0.25- 0.99), CLOCK rs3749474 (OR 0.56, 95\% CI 0.31- 0.99); ROR-b rs3903529 (OR 0.57, 95\% CI 0.34- 0.95), ROR-b rs7022435 (OR 0.16, 95\% CI 0.05- 0.55); ROR-b rs7022435 (OR 0.17, 95\% CI 0.03- 0.84); ROR-b rs3750420 (OR 0.40, 95\% CI $0.18-0.88$ ) and MTNR1A rs 13113549 (OR 0.50, 95\% CI 0.28-0.90).

The cases and controls were then grouped according to both intensity and duration of night work, that is, 
Table 1 Characteristics of controls and breast cancer cases.

\begin{tabular}{|c|c|c|c|}
\hline Parameter & Cases $(n=563)$ & Controls $(n=619)$ & $P$ value \\
\hline Age $(y r)^{a}$ & & & $0.51^{\mathrm{b}}$ \\
\hline Mean (SD) & $52.40(7.88)$ & $52.37(8.18)$ & \\
\hline Median & 50 & 55 & \\
\hline $\operatorname{Min} / \max$ & $34 / 70$ & $34 / 70$ & \\
\hline \multicolumn{4}{|l|}{ Selection period } \\
\hline 1996 to $1999 / 2000$ to $2007(n)$ & $195 / 368$ & $221 / 398$ & $0.72^{c}$ \\
\hline Breast cancer in first-degree family ${ }^{d}(n)$ & 557 & 615 & \\
\hline Yes/no & $104 / 453$ & $54 / 561$ & $<0.001^{c}$ \\
\hline Hormone therapy in past $2 \mathrm{yr}^{\mathrm{e}}(n)$ & 552 & 605 & \\
\hline Yes/no & $127 / 425$ & $121 / 484$ & $0.22^{c}$ \\
\hline Alcohol use ${ }^{f}$ & $n=563$ & $n=619$ & \\
\hline Yes/no & $43 / 520$ & $37 / 582$ & $0.26^{c}$ \\
\hline Children (n) & 563 & 619 & \\
\hline $0 / \geq 1$ & $77 / 486$ & $81 / 538$ & $0.80^{c}$ \\
\hline
\end{tabular}

${ }^{\mathrm{a}}$ Age at selection for controls and age at diagnosis for cases. ${ }^{\mathrm{b}}$ Derived from Mann-Whitney $U$-test for two independent samples (two-sided) assuming the medians are the same across cases and controls. 'Pearson's $\chi^{2}$ test (two-sided). ${ }^{d}$ Cancer in sister or mother. ${ }^{e}$ Hormone therapy among postmenopausal women only. ${ }^{f}$ Alcohol consumption at least twice weekly.

maximum number of consecutive nights they had ever worked for at least $5 \mathrm{yr}$. In subjects with at least four consecutive night shifts, increased risk of breast cancer was associated with variant alleles of BMAL1 (rs2290035 (OR 1.91, 95\% CI 1.08- 3.37; and OR 1.95, 95\% CI 0.973.90) and rs969485 (OR 1.64, 95\% CI 1.03- 2.61; and OR $1.63,95 \%$ CI $0.96-2.76)$ ) and $R O R-b(\operatorname{rs} 3750420$ (OR 1.60, 95\% CI 1.0- 2.56), rs3903529 (OR 2.77, 95\% CI 0.98- 7.86)) and MTNR1B rs10830963 (OR 9.12, 95\% CI 1.14- 73.09). In the group with three consecutive night shifts, the SNP rs11695472 in the PER2 gene (OR $2.69,95 \%$ CI 1.08- 6.73) was associated with increased risk of breast cancer. The SNPs BMAL1 rs2278749 (OR 0.51, 95\% CI 0.29- 0.87), BMAL2 rs2306074 (OR 0.30, 95\% CI 0.17- 0.75), CLOCK rs3749474 (OR 0.24, 95\% CI 0.05 to 1.18 ), CSNK1E (rs1534891 (OR 0.55, 95\% CI 0.30- 1.01), rs5757037 (OR 0.67, 95\% CI 0.45- 0.99)),
NPAS2 rs17024926 (OR 0.33, 95\% CI 0.13- 0.84; and OR 0.24, 95\% CI 0.09- 0.67); PER3 rs1012477 (OR 0.42, 0.24- 0.72; and OR 0.38, 95\% CI 0.18- 0.66), ROR-b (rs7022435 (OR 0.25, 95\% CI 0.07- 0.89), rs3750420 (OR 0.37, 95\% CI 0.14- 0.96; and OR 0.33, 95\% CI 0.11- 1.0)) and MTNR1A rs131113549 (OR 0.47, 95\% CI 0.25 0.91 ) were associated with decreased breast cancer risk (Table 5).

After applying FPRP and BFDP tests, in subjects with four or more night shifts (Table 4), increased risk of breast cancer associated with homozygote carriers of the variant alleles of SNPs rs3760138 and rs4238989 in the AANAT gene was found to be noteworthy. In subjects with three consecutive night shifts, the odds of reduced risk of breast cancer associated with carriage of variant alleles of SNPs in CLOCK (rs3749474), ROR-b (rs3903529, rs3750420) and MTNR1A (rs131113549) were found noteworthy.

Table 2 Occupational exposure data and number of cases and controls in each exposure group.

\begin{tabular}{|c|c|c|}
\hline Maximum number of consecutive night shifts in all jobs that nurse has worked ${ }^{\mathrm{a}}$ & $\begin{array}{l}\text { No. of } \\
\text { cases }^{\text {b }}\end{array}$ & $\begin{array}{l}\text { No. of } \\
\text { controls }\end{array}$ \\
\hline Only day work or one or two consecutive night shifts (reference) & $170(227)$ & $186(275)$ \\
\hline Three consecutive night shifts & $156(181)$ & $189(272)$ \\
\hline Four or more consecutive night shifts & $239(291)$ & $244(348)$ \\
\hline Total & $563(699)$ & $619(895)$ \\
\hline \multicolumn{3}{|c|}{$\begin{array}{l}\text { Intensity of night work: Maximum number of consecutive night shifts the nurse has ever worked for } 5 \text { or } \\
\text { more yearsc }\end{array}$} \\
\hline Only day work or one or two consecutive nights for 5 or more years (reference) & $326(421)$ & $362(524)$ \\
\hline Three consecutive night shifts for 5 or more years & $125(147)$ & $154(227)$ \\
\hline Four or more consecutive night shifts for 5 or more years & $112(131)$ & $103(144)$ \\
\hline Total & $563(699)$ & $619(895)$ \\
\hline
\end{tabular}

${ }^{a}$ Maximum number of consecutive night shifts in all jobs: 0 to 2, 3 or $\geq 4$. ${ }^{b}$ Number of nurses who had consented to send saliva for genetic analysis (numbers in parentheses show the total number of cases and controls interviewed in each exposure group). 'Maximum number of consecutive night shifts the nurse has ever worked for 5 or more years. 
Table 3 Odds ratios and $95 \%$ confidence intervals for polymorphisms with statistically significant associations with risk of breast cancer.

\begin{tabular}{|c|c|c|c|c|}
\hline \multirow{2}{*}{$\begin{array}{l}\text { Genes and SNPs } \\
B M A L 1\end{array}$} & \multicolumn{2}{|c|}{ Frequency of genotypes ${ }^{a}$} & \multirow[t]{2}{*}{ OR $(95 \% \mathrm{Cl})^{\mathrm{b}} P^{c}$} & \multirow[t]{2}{*}{ FPRP $^{d}$ BFDP $^{\mathrm{e}}$} \\
\hline & No. of cases & No. of controls & & \\
\hline \multicolumn{5}{|l|}{ rs2278749 } \\
\hline CC & 375 & 381 & 1.0 & \\
\hline CT & 141 & 164 & $0.93(0.70-1.22)$ & \\
\hline \multirow[t]{2}{*}{$\pi$} & 13 & 30 & $0.45(0.23-0.90) 0.02$ & 0.160 .21 \\
\hline & & & $P_{\text {trend }}=0.07$ & \\
\hline \multicolumn{5}{|l|}{ CLOCK } \\
\hline \multicolumn{5}{|l|}{ rs3749474 } \\
\hline CC & 223 & 228 & 1.0 & \\
\hline CT & 251 & 260 & $1.01(0.78-1.31)$ & \\
\hline \multirow[t]{2}{*}{$\pi$} & 68 & 108 & $0.64(0.45-0.92) 0.02$ & 0.140 .11 \\
\hline & & & $P_{\text {trend }}=\mathbf{0 . 0 3}$ & \\
\hline \multicolumn{5}{|l|}{ AANAT } \\
\hline \multicolumn{5}{|l|}{ rs3760138 } \\
\hline$\pi$ & 149 & 181 & 1.0 & \\
\hline TG & 269 & 315 & $1.00(0.75-1.32)$ & \\
\hline \multirow[t]{2}{*}{ GG } & 128 & 106 & $1.42(1.01-2.01) 0.05$ & 0.130 .18 \\
\hline & & & $P_{\text {trend }}=0.07$ & \\
\hline \multicolumn{5}{|l|}{ rs4238989 } \\
\hline GG & 161 & 203 & 1.0 & \\
\hline GC & 263 & 288 & $1.13(0.86-1.49)$ & \\
\hline \multirow[t]{2}{*}{ CC } & 120 & 104 & $1.43(1.01-2.01) 0.04$ & 0.110 .16 \\
\hline & & & $P_{\text {trend }}=0.13$ & \\
\hline
\end{tabular}

${ }^{a}$ Number of cases and controls with successful genotyping data. ${ }^{b}$ Odds ratios (ORs) derived from unconditional logistic regression and adjusted for age at diagnosis (cases) or day of reference (controls), selection period, alcohol use, hormone replacement therapy for the past 2 years, number of children and history of breast cancer in first-degree family (mother or sister). 'Pearson's $\chi^{2}$ test (two-sided) comparing frequency of the homozygote variant genotypes with homozygote common genotypes (reference). ${ }^{\mathrm{d}}$ The false-positive report probability (FPRP) was calculated as described by Wacholder et al. [42]. FPRP < 0.2 was considered noteworthy. ${ }^{\text {T}}$ The Bayesian false-discovery probability (BFDP) was calculated as described by Wakefield et al. [41]. BFDP < 0.8 was considered noteworthy. For more details on FPRP and BFDP, see Methods section. $P_{\text {trend }}$ values for the Cochran-Armitage trend test were calculated from $2 \times C$ tables using frequency of cases and controls.

With respect to both intensity and duration of night shift work for at least $5 \mathrm{yr}$ (Table 5), in subjects with at least four consecutive night shifts, increased risk of breast cancer was associated with variant alleles of BMAL1 (rs2290035, rs969485) and ROR-b (rs3750420). In the subjects with three night shifts, SNPs in BMAL1 (rs2278749), BMAL2 (rs2306074), CSNK1E (rs5757037), NPAS2 (rs17024926), PER3 (rs1012477) and MTNR1A (rs131113549) were associated with decreased breast cancer risk. Haplotype analysis of the polymorphisms localized on the same chromosome did not result in any significant associations (data not shown).

\section{Discussion}

In the main effects analysis, two SNPs in BMAL1 and $C L O C K$ were associated with risk of breast cancer. There are no functional data to explain how each SNP in each of the genes may affect the risk for breast cancer. SNPs may change transcription binding affinity of the BMAL1/ CLOCK complex that is necessary for expression of the target genes. For example, a reduced affinity of BMAL1/
CLOCK to CLOCK promoter due to polymorphisms in these genes may lead to lower expression of CLOCK proteins. A reduction in the levels of another core circadian protein (CRY2) in breast epithelial cells in culture has been shown to lead to higher accumulation of DNA damage after exposure to chemical mutagens $[48,49]$. Downregulation of CLOCK has also been reported to cause alterations in expression of genes involved in breast carcinogenesis [50].

Several SNPs in the core circadian genes had noteworthy associations with breast cancer risk. Women with the highest number of successive night shifts and carrying at least one variant allele of SNPs in the two core circadian genes BMAL1 (rs2290035, rs969485) and ROR-b (rs3903529, rs3750420) had a noteworthy increased risk of breast cancer. Although not noteworthy, there was also a 2.75 increased risk of breast cancer in women who had worked four or more night shifts and carrying two variant G alleles of CLOCK rs111133373 compared to women with the same genotype in the reference group. Interestingly, women with three successive night shifts and 
Table 4 Odds ratios and $95 \%$ confidence intervals comparing odds of breast cancer associated with each polymorphism among women with less than two consecutive night shifts compared to women with three consecutive night shifts or women with four or more consecutive night shifts.

\begin{tabular}{|c|c|c|c|c|c|c|c|c|}
\hline \multirow[t]{3}{*}{ Genes and SNPs } & \multicolumn{8}{|c|}{ Number of consecutive night shifts that nurse has worked in all jobs } \\
\hline & \multicolumn{2}{|c|}{$\begin{array}{l}0 \text { to } 2 \text { consecutive night } \\
\text { shifts }\end{array}$} & \multicolumn{3}{|c|}{ Three consecutive night shifts } & \multicolumn{3}{|c|}{$\geq 4$ consecutive night shifts } \\
\hline & $\mathrm{Ca}^{\mathrm{a}} / \mathrm{Co}^{\mathrm{a}}$ & OR $(95 \% \mathrm{Cl})^{\mathrm{b}}$ & $\mathrm{Ca}^{\mathrm{a}} / \mathrm{Co}^{\mathrm{a}}$ & OR $(95 \% \mathrm{Cl})^{\mathrm{b}} P_{\text {inter }}^{\mathrm{c}}$ & FPRPd $^{\mathrm{d}}$ BFDP $^{\mathrm{e}}$ & $\mathrm{Ca}^{\mathrm{a}} / \mathrm{Co}^{\mathrm{a}}$ & OR $(95 \% \mathrm{Cl})^{\mathrm{b}} P_{\text {inter }}{ }^{\mathrm{c}}$ & FPRP $^{d}$ BFDP \\
\hline \multicolumn{9}{|l|}{$\overline{C L O C K}$} \\
\hline \multicolumn{9}{|l|}{ rs11133373 } \\
\hline CC & $72 / 77$ & 1.0 (reference) & $71 / 68$ & $0.82(0.52-1.30)$ & & $103 / 99$ & $1.29(0.79-2.11)$ & \\
\hline CG & $76 / 72$ & $0.98(0.61-1.57)$ & $58 / 82$ & $0.77(0.53-1.13)$ & & $93 / 114$ & $0.87(0.63-1.20)$ & \\
\hline \multirow[t]{2}{*}{ GG } & $14 / 25$ & $1.05(0.49-2.29)$ & $13 / 29$ & $0.50(0.25-0.99) 0.05$ & 0.220 .23 & $28 / 23$ & $1.17(0.65-2.10)$ & \\
\hline & & & & $P_{\text {trend }}{ }^{\mathrm{f}}=\mathbf{0 . 0 4}$ & & & $P_{\text {trend }}{ }^{\mathrm{f}}=0.95$ & \\
\hline CC & $72 / 77$ & 1.0 (reference) & $71 / 68$ & $0.82(0.52-1.30)$ & & $103 / 99$ & $1.29(0.79-2.11)$ & \\
\hline CG & $76 / 72$ & 1.0 (reference) & $58 / 82$ & $0.88(0.56-1.39)$ & & $93 / 114$ & $0.79(0.48-1.30)$ & \\
\hline GG & $14 / 25$ & 1.0 (reference) & $13 / 29$ & $0.83(0.34-2.04)$ & & $28 / 23$ & $2.75(1.07-7.09) 0.04$ & 0.300 .25 \\
\hline \multicolumn{9}{|l|}{ rs3749474 } \\
\hline CC & $67 / 71$ & 1.0 (reference) & $60 / 66$ & $0.95(0.57-1.57)$ & & $96 / 91$ & $1.14(0.72-1.80)$ & \\
\hline $\mathrm{CT}$ & $77 / 76$ & $1.12(0.69-1.81)$ & $73 / 78$ & $1.01(0.70-1.46)$ & & 101/106 & $1.03(0.74-1.42)$ & \\
\hline \multirow[t]{2}{*}{$\Pi$} & $16 / 30$ & $0.55(0.27-1.14)$ & $20 / 37$ & $0.56(0.31-0.99) 0.05$ & 0.180 .21 & $32 / 41$ & $0.85(0.52-1.39)$ & \\
\hline & & & & $P_{\text {trend }}{ }^{f}=0.30$ & & & $P_{\text {trend }}{ }^{\mathrm{f}}=0.54$ & \\
\hline CC & $67 / 71$ & 1.0 (reference) & $60 / 66$ & $0.95(0.57-1.57)$ & & $96 / 91$ & $1.14(0.72-1.80)$ & \\
\hline$C T$ & $77 / 76$ & 1.0 (reference) & $73 / 78$ & $0.93(0.58-1.48)$ & & 101/106 & $0.98(0.63-1.51)$ & \\
\hline$\pi$ & $16 / 30$ & 1.0 (reference) & $20 / 37$ & $1.10(0.46-2.61)$ & & $32 / 41$ & $1.35(0.61-3.00)$ & \\
\hline \multicolumn{9}{|l|}{ CRY2 } \\
\hline \multicolumn{9}{|l|}{ rs11038689 } \\
\hline AA & $87 / 97$ & 1.0 (reference) & $84 / 97$ & $0.99(0.67-1.47)$ & & $127 / 127$ & $1.36(0.87-2.13)$ & \\
\hline AG & $52 / 55$ & $1.10(0.66-1.81)$ & $45 / 57$ & $0.82(0.54-1.26)$ & & $68 / 70$ & $0.94(0.65-1.37)$ & \\
\hline \multirow[t]{2}{*}{ GG } & $8 / 11$ & $0.86(0.32-2.28)$ & $13 / 12$ & $1.11(0.49-2.51)$ & & $7 / 20$ & $0.42(0.17-1.01) 0.05$ & 0.310 .25 \\
\hline & & & & $P_{\text {trend }}^{f}=0.91$ & & & $P_{\text {trend }}{ }^{f}=0.39$ & \\
\hline AA & $87 / 97$ & 1.0 (reference) & $84 / 97$ & $0.99(0.67-1.47)$ & & $127 / 127$ & $1.36(0.87-2.13)$ & \\
\hline AG & $52 / 55$ & 1.0 (reference) & $45 / 57$ & $0.98(0.58-1.68)$ & & $68 / 70$ & $0.82(0.46-1.47)$ & \\
\hline GG & $8 / 11$ & 1.0 (reference) & $13 / 12$ & $0.44(0.09-2.06)$ & & $7 / 20$ & $1.44(0.33-6.20)$ & \\
\hline \multicolumn{9}{|l|}{ rs1401417 } \\
\hline CC & $78 / 94$ & 1.0 (reference) & $75 / 95$ & $1.01(0.65-1.58)$ & & $119 / 120$ & $1.27(0.84-1.91)$ & \\
\hline CG & $46 / 54$ & $1.10(0.65-1.86)$ & $40 / 56$ & $0.76(0.49-1.18)$ & & $72 / 68$ & $1.13(0.77-1.64)$ & \\
\hline \multirow[t]{2}{*}{ GG } & $8 / 8$ & $1.36(0.47-3.94)$ & $10 / 13$ & $0.86(0.37-2.01)$ & & $4 / 17$ & $0.31(0.10-0.94) 0.04$ & 0.370 .26 \\
\hline & & & & $P_{\text {trend }}{ }^{f}=0.61$ & & & $P_{\text {trend }}{ }^{f}=0.78$ & \\
\hline CC & $78 / 94$ & 1.0 (reference) & $75 / 95$ & $1.01(0.65-1.58)$ & & $119 / 120$ & $1.27(0.84-1.91)$ & \\
\hline CG & $46 / 54$ & 1.0 (reference) & $40 / 56$ & $0.76(0.42-1.37)$ & & $72 / 68$ & $1.11(0.65-1.90)$ & \\
\hline GG & $8 / 8$ & 1.0 (reference) & $10 / 13$ & 0.22 (0.18 to 3.74$)$ & & $4 / 17$ & 0.25 (0.05 to 1.37$)$ & \\
\hline \multicolumn{9}{|l|}{ NPAS2 } \\
\hline \multicolumn{9}{|l|}{ rs2305160 } \\
\hline GG & $75 / 72$ & 1.0 (reference) & $58 / 71$ & $0.78(0.48-1.28)$ & & $105 / 90$ & $1.16(0.74-1.81)$ & \\
\hline GA & $61 / 74$ & $0.82(0.50-1.33)$ & $69 / 83$ & $0.87(0.60-1.25)$ & & $94 / 104$ & $0.93(0.67-1.29)$ & \\
\hline \multirow[t]{2}{*}{ AA } & $25 / 27$ & $0.89(0.46-1.72)$ & $21 / 28$ & $0.73(0.40-1.34)$ & & $27 / 35$ & $0.87(0.51-1.50)$ & \\
\hline & & & & $P_{\text {trend }}{ }^{\mathrm{f}}=0.26$ & & & $P_{\text {trend }}{ }^{f}=0.21$ & \\
\hline GG & $75 / 72$ & 1.0 (reference) & $58 / 71$ & $0.78(0.48-1.28)$ & & $105 / 90$ & $1.16(0.74-1.81)$ & \\
\hline GA & $61 / 74$ & 1.0 (reference) & $69 / 83$ & $1.06(0.65-1.73)$ & & $94 / 104$ & $1.12(0.71-1.76)$ & \\
\hline AA & $25 / 27$ & 1.0 (reference) & $21 / 28$ & $0.76(0.33-1.72)$ & & $27 / 35$ & $0.86(0.39-1.92)$ & \\
\hline \multicolumn{9}{|l|}{$R O R-b$} \\
\hline \multicolumn{9}{|l|}{ rs3903529 } \\
\hline$\pi$ & $76 / 100$ & 1.0 (reference) & $81 / 89$ & $1.29(0.82-2.01)$ & & $120 / 111$ & $1.41(0.93-2.14)$ & \\
\hline TA & $72 / 58$ & $1.67(1.03-2.70)$ & $51 / 73$ & $0.74(0.49-1.10)$ & & $81 / 109$ & $0.81(0.58-1.13)$ & \\
\hline
\end{tabular}


Table 4 Odds ratios and $95 \%$ confidence intervals comparing odds of breast cancer associated with each polymorphism among women with less than two consecutive night shifts compared to women with three consecutive night shifts or women with four or more consecutive night shifts. (Continued)

\begin{tabular}{|c|c|c|c|c|c|c|c|c|}
\hline$\overline{A A}$ & $10 / 16$ & $0.89(0.37-2.15)$ & $12 / 19$ & $\begin{array}{l}0.61(0.29-1.31) \\
P_{\text {trend }}{ }^{f}=0.60\end{array}$ & & $27 / 15$ & $\begin{array}{l}1.87(1.00-3.61) 0.06 \\
P_{\text {trend }} f=0.28\end{array}$ & 0.240 .24 \\
\hline$\pi$ & $76 / 100$ & 1.0 (reference) & $81 / 89$ & $1.29(0.82-2.01)$ & & $120 / 111$ & $1.41(0.93-2.14)$ & \\
\hline TA & $72 / 58$ & 1.0 (reference) & $51 / 73$ & $0.57(0.34-0.95) 0.03$ & 0.120 .18 & $81 / 109$ & $0.66(0.41-1.05)$ & \\
\hline AA & $10 / 16$ & 1.0 (reference) & $12 / 19$ & $0.91(0.30-2.80)$ & & $27 / 15$ & $\begin{array}{l}2.47(0.83-7.34) \\
\boldsymbol{P}_{\text {trend }}=\mathbf{0 . 0 3}\end{array}$ & \\
\hline \multicolumn{9}{|c|}{ rs7022435 } \\
\hline GG & $89 / 100$ & 1.0 (reference) & $92 / 101$ & $1.06(0.69-1.62)$ & & $124 / 134$ & $1.06(0.71-1.57)$ & \\
\hline GA & $65 / 62$ & $1.21(0.75-1.93)$ & $53 / 63$ & $0.93(0.61-1.40)$ & & $82 / 84$ & $1.05(0.75-1.49)$ & \\
\hline AA & $8 / 9$ & $1.12(0.41-3.06)$ & $3 / 18$ & $\begin{array}{l}\mathbf{0 . 1 6}(\mathbf{0 . 0 5}-\mathbf{0 . 5 5}) \mathbf{0 . 0 0 4} \\
P_{\text {trend }}{ }^{f}=0.21\end{array}$ & 0.240 .23 & $18 / 12$ & $\begin{array}{l}1.55(0.73-3.30) \\
P_{\text {trend }}{ }^{f}=0.31\end{array}$ & \\
\hline \multicolumn{9}{|c|}{ rs7022435 } \\
\hline GG & $89 / 100$ & 1.0 (reference) & $92 / 101$ & $1.06(0.69-1.62)$ & & $124 / 134$ & $1.06(0.71-1.57)$ & \\
\hline GA & $65 / 62$ & 1.0 (reference) & $53 / 63$ & $0.81(0.48-1.37)$ & & $82 / 84$ & $0.93(0.58-1.49)$ & \\
\hline AA & $8 / 9$ & 1.0 (reference) & $3 / 18$ & $0.17(0.03-0.84) 0.03$ & 0.490 .28 & $18 / 12$ & $1.68(0.44-6.33)$ & \\
\hline \multicolumn{9}{|c|}{ rs3750420 } \\
\hline CC & $68 / 87$ & 1.0 (reference) & $68 / 80$ & $1.12(0.70-1.81)$ & & $93 / 112$ & $1.03(0.66-1.61)$ & \\
\hline $\mathrm{CT}$ & $76 / 72$ & $1.35(0.84-2.18)$ & $68 / 72$ & $1.11(0.76-1.62)$ & & 100/92 & $1.32(0.95-1.83)$ & \\
\hline$\Pi$ & $13 / 15$ & $1.25(0.54-2.87)$ & $9 / 25$ & $\begin{array}{l}\mathbf{0 . 4 0}(\mathbf{0 . 1 8}-\mathbf{0 . 8 8}) \mathbf{0 . 0 2} \\
P_{\text {trend }}{ }^{f}=0.58\end{array}$ & 0.190 .22 & $29 / 21$ & $\begin{array}{l}1.57(0.87-2.83) \\
P_{\text {trend }}{ }^{f}=0.04\end{array}$ & \\
\hline CC & $68 / 87$ & 1.0 (reference) & $68 / 80$ & $1.12(0.70-1.81)$ & & $93 / 112$ & $1-03(0.66-1.61)$ & \\
\hline$C T$ & $76 / 72$ & 1.0 (reference) & $68 / 72$ & $0.91(0.56-1.48)$ & & 100/92 & $1.13(0.72-1.76)$ & \\
\hline$\pi$ & $13 / 15$ & 1.0 (reference) & $9 / 25$ & $0.34(0.11-1.05)$ & & $29 / 21$ & $1.35(0.51-3.58)$ & \\
\hline \multicolumn{9}{|c|}{ AANAT } \\
\hline \multicolumn{9}{|c|}{ rs3760138 } \\
\hline$\pi$ & $38 / 52$ & 1.0 (reference) & $42 / 57$ & $0.93(0.51-1.70)$ & & $69 / 72$ & $1.19(0.68-2.08)$ & \\
\hline TG & $86 / 85$ & $1.17(0.68-2.00)$ & $75 / 95$ & $0.87(0.61-1.24)$ & & 108/135 & $0.91(0.67-1.24)$ & \\
\hline GG & $40 / 42$ & $1.18(0.63-2.21)$ & $33 / 32$ & $\begin{array}{l}1.23(0.72-2.08) \\
P_{\text {trend }}\end{array}$ & & $55 / 32$ & $\begin{array}{l}1.82(1.13-2.93) 0.01 \\
P_{\text {trend }}=0.04\end{array}$ & 0.060 .14 \\
\hline$\Pi$ & $38 / 52$ & 1.0 (reference) & $42 / 57$ & $0.93(0.51-1.70)$ & & $69 / 72$ & $1.19(0.68-2.08)$ & \\
\hline TG & $86 / 85$ & 1.0 (reference) & $75 / 95$ & $0.80(0.51-1.25)$ & & $108 / 135$ & $0.82(0.54-1.23)$ & \\
\hline GG & $40 / 42$ & 1.0 (reference) & $33 / 32$ & $1.28(0.64-2.57)$ & & $55 / 32$ & $1.84(0.94-3.62)$ & \\
\hline \multicolumn{9}{|c|}{ rs4238989 } \\
\hline GG & $43 / 60$ & 1.0 (reference) & $50 / 59$ & $1.13(0.64-2.01)$ & & $68 / 84$ & $1.07(0.62-1.83)$ & \\
\hline GC & $77 / 82$ & $1.20(0.72-2.02)$ & $66 / 86$ & $0.87(0.60-1.27)$ & & $120 / 120$ & $1.18(0.87-1.61)$ & \\
\hline CC & $40 / 35$ & $1.47(0.79-2.74)$ & $37 / 39$ & $\begin{array}{l}1.15(0.70-1.88) \\
P_{\text {trend }}{ }^{f}=0.49\end{array}$ & & $43 / 30$ & $\begin{array}{l}1.62(1.00-2.68) 0.06 \\
P_{\text {trend }}{ }^{f}=0.02\end{array}$ & 0.190 .22 \\
\hline GG & $43 / 60$ & 1.0 (reference) & $50 / 59$ & $1.13(0.64-2.01)$ & & $68 / 84$ & $1.07(0.62-1.83)$ & \\
\hline GC & $77 / 82$ & 1.0 (reference) & $66 / 86$ & $0.85(0.53-1.37)$ & & $120 / 120$ & $1.11(0.73-1.68)$ & \\
\hline CC & $40 / 35$ & 1.0 (reference) & $37 / 39$ & $1.02(0.52-2.01)$ & & $43 / 30$ & $1.40(0.69-2.83)$ & \\
\hline \multicolumn{9}{|c|}{ MTNR1A } \\
\hline \multicolumn{9}{|c|}{ rs13113549 } \\
\hline$\pi$ & $51 / 60$ & 1.0 (reference) & $64 / 58$ & $1.36(0.79-2.34)$ & & $81 / 78$ & $1.37(0.82-2.27)$ & \\
\hline TC & $84 / 88$ & $1.19(0.72-1.96)$ & $67 / 80$ & $0.89(0.61-1.30)$ & & $102 / 96$ & $1.08(0.78-1.50)$ & \\
\hline CC & $26 / 29$ & $1.12(0.57-2.20)$ & $20 / 42$ & $\begin{array}{l}\mathbf{0 . 5 0}(\mathbf{0 . 2 8}-\mathbf{0 . 9 0}) \mathbf{0 . 0 2} \\
P_{\text {trend }}{ }^{f}=0.11\end{array}$ & 0.110 .18 & $47 / 56$ & $\begin{array}{l}0.82(0.54-1.26) \\
P_{\text {trend }}{ }^{f}=0.95\end{array}$ & \\
\hline$\pi$ & $51 / 60$ & 1.0 (reference) & $64 / 58$ & $1.36(0.79-2.34)$ & & $81 / 78$ & $1.37(0.82-2.27)$ & \\
\hline TC & $84 / 88$ & 1.0 (reference) & $67 / 80$ & $0.88(0.55-1.40)$ & & 102/96 & $1.11(0.73-1.71)$ & \\
\hline CC & $26 / 29$ & 1.0 (reference) & $20 / 42$ & $0.55(0.25-1.23)$ & & $47 / 56$ & $0.79(0.40-1.59)$ & \\
\hline
\end{tabular}


Table 4 Odds ratios and $95 \%$ confidence intervals comparing odds of breast cancer associated with each polymorphism among women with less than two consecutive night shifts compared to women with three consecutive night shifts or women with four or more consecutive night shifts. (Continued)

\begin{tabular}{|c|c|c|c|c|c|c|}
\hline CC & 95/97 & 1.0 (reference) & $76 / 103$ & $0.74(0.48-1.13)$ & $119 / 129$ & $0.90(0.61-1.34)$ \\
\hline CG & $56 / 69$ & $0.74(0.46-1.19)$ & $55 / 60$ & $1.04(0.69-1.56)$ & $86 / 90$ & $1.06(0.76-1.49)$ \\
\hline GG & $7 / 8$ & $0.76(0.25-2.32)$ & $15 / 13$ & $\begin{array}{l}1.29(0.59-2.81) \\
P_{\text {trend }}{ }^{f}=0.99\end{array}$ & $19 / 8$ & 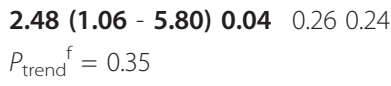 \\
\hline CC & 95/97 & 1.0 (reference) & 76/103 & $0.74(0.48-1.13)$ & 119/129 & $0.90(0.61-1.34)$ \\
\hline$C G$ & $56 / 69$ & 1.0 (reference) & $55 / 60$ & $1.18(0.70-2.00)$ & $86 / 90$ & $1.23(0.76-1.97)$ \\
\hline GG & $7 / 8$ & 1.0 (reference) & $15 / 13$ & $1.55(0.38-6.37)$ & $19 / 8$ & $3.51(0.73-16.96)$ \\
\hline
\end{tabular}

${ }^{a}$ Number of cases and controls that were successfully genotyped. ${ }^{b}$ OR derived from unconditional logistic regression, including an interaction term between the night shift variable and the SNP genotypes and adjusted for age at diagnosis (cases) or interview (controls), selection period, alcohol use, hormone replacement therapy for the past 2 years, number of children and history of breast cancer in first-degree family members (mother or sister). ${ }^{c} P$ interaction was obtained from the interaction term between the SNP genotypes and the night shift variable using frequency of the common genotype in the 0 to 2 night shift group as reference. Only significant or near-significant values are shown. ${ }^{\mathrm{d}}$ The false-positive report probability (FPRP) was calculated as described by Wacholder et al. [42].

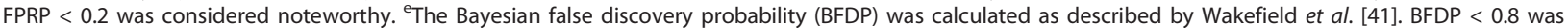
considered noteworthy. For more details on FPRP and BFDP, see the Methods section. ${ }^{\mathrm{f}} P_{\text {trend }}$ values for Cochran-Armitage trend test were calculated from $2 \times C$ tables using frequency of genotypes in cases and controls from 0 to 2 night shift group as reference.

carrying the same SNPs and genotypes had a reduced risk of breast cancer, indicating a protective role for these genetic variants. In addition, women in this exposure group carrying at least one variant allele of several SNPs in the other core circadian genes such as BMAL2, CSNK1E, NPAS2 and PER3 had a noteworthy reduced risk of breast cancer. For comparison, only two SNPs in the CRY2 gene (rs11038689 and rs1401417) had protective effects in women with four or more night shifts, though this effect was not noteworthy.

Many of the associations, such as PER3 (rs1012477), CSNK1E (rs1534891) and CLOCK (rs11133373, rs3749474), are novel in relation to breast risk but may not be specific to breast cancer, because they have also been found to be associated with prostate and colorectal cancer risk [47,51]. It might be that these SNPs affect expression of the respective genes. Current results from the available literature on the functionality of other SNPs in these genes are not conclusive. For example, it has previously been seen that, in healthy nurses and midwives who worked either day or rotating night shifts, there were no differences between BMAL1, CLOCK, CRY1, CRY2, PER1, PER2 and PER3 gene expression levels in peripheral blood leukocytes [52]. In the same subjects, however, polymorphisms of the circadian genes BMAL1 (rs2279287), CLOCK (rs1801260), PER1 (rs2735611), PER2 (rs2304672), PER3 (rs10462020), CRY1 (rs8192440) and CRY2 (rs10838527, rs10838527) were not found to be different between shift workers and day-only workers [53].

In a recent study by Monsees et al. [54], the SNP rs2305160 (Ala394Thr) in the NPAS2 gene was found to be associated with reduced breast cancer risk in women with less than 2 yr of shift work. We were not able to confirm such an association in our study; however, for another SNP, rs17024926 in the NPAS2 gene, we found a reduced risk of breast cancer in shift workers who had worked three night shifts for at least $5 \mathrm{yr}$ (Table 5). The reduced risk was associated with the variant homozygote genotype where carriers of the $\mathrm{CC}$ variant genotype had reduced risk of breast cancer compared to subjects who had worked no or few night shifts carrying either the variant CC genotypes or the common TT genotype (Table 5). The NPAS2 rs17024926 has been found to be associated with risk of prostate cancer [47]. This SNP was not genotyped in the study by Monsees et al. [54]; however, the reasons why the results of our study and those of Monsees et al. differ for Ala394Thr variant are not known. This difference could not be due to differences in MAF in the two populations, first because the MAF 0.37 for this SNP in our population (Additional File 2, Table S2) is similar to the MAF 0.34 in the study by Monsees et al., and second because we regenotyped this SNP in our subjects and again found no associations. There could be several other possibilities, such as differences in study design, population differences in incidence of breast cancer, exposure metrics and the fact that breast cancer is known to be a genetically heterogeneous disease. Nevertheless, both studies support a role of circadian genes in breast cancer in shift workers, which warrants further confirmation and validation in future studies.

Increased risk of breast cancer in women with four or more night shifts was associated with ROR- $b$ SNPs rs3903529 and rs3750420 (heterozygotes), whereas women with three night shifts and carrying a homozygote variant of rs7022435 and rs3750420 had reduced risk. The three SNPs are located in intron 1 downstream of the exon 1 in the ROR- $b$ transcripts. These SNPs are part of a haplotype block that spans the region containing the promoter region and the exon 1 (55 kb upstream to $6 \mathrm{~kb}$ downstream) of the gene. This haplotype has also been associated with an increased risk of human bipolar syndrome [55], but we were not able to find such an association with 
Table 5 Odds ratios and $95 \%$ confidence intervals comparing odds of breast cancer associated with each polymorphism among women with less than two consecutive night shifts for 5 or more years compared to women with three consecutive night shifts or women with four or more consecutive night shifts for $\mathbf{5}$ or more years

\begin{tabular}{|c|c|c|c|c|c|c|c|c|}
\hline \multirow{3}{*}{$\begin{array}{l}\text { Genes and } \\
\text { SNPs }\end{array}$} & \multicolumn{8}{|c|}{ Number of consecutive night shifts nurse has ever worked for 5 or more years } \\
\hline & \multicolumn{2}{|c|}{$\begin{array}{c}0 \text { to } 2 \text { consecutive } \\
\text { nights }\end{array}$} & \multicolumn{3}{|c|}{3 consecutive nights } & \multicolumn{3}{|c|}{4 or more consecutive nights } \\
\hline & $\begin{array}{l}\mathrm{Ca}^{\mathrm{a} /} \\
\mathrm{Co}^{\mathrm{a}}\end{array}$ & OR $(95 \% \mathrm{Cl})^{\mathrm{b}}$ & $\begin{array}{l}\mathrm{Ca}^{\mathrm{a} /} \\
\mathrm{Co}^{\mathrm{a}}\end{array}$ & OR $(95 \% \mathrm{Cl})^{\mathrm{b}} P_{\text {inter }}{ }^{\mathrm{c}}$ & $\begin{array}{l}\text { FPRP }^{d} \\
\text { BFDP }^{e}\end{array}$ & $\begin{array}{l}\mathrm{Ca}^{\mathrm{a}} / \\
\mathrm{Co}^{\mathrm{a}}\end{array}$ & OR $(95 \% \mathrm{Cl})^{\mathrm{b}} P_{\text {inter }}^{\mathrm{c}}$ & $\begin{array}{l}\text { FPRP }^{d} \\
\text { BFDP }^{e}\end{array}$ \\
\hline \multicolumn{9}{|l|}{$\begin{array}{l}\text { ARNTL1/ } \\
\text { BMAL1 }\end{array}$} \\
\hline \multicolumn{9}{|l|}{ rs2290035 } \\
\hline$\pi$ & $106 / 121$ & 1.0 (reference) & $40 / 46$ & $0.98(0.58-1.65)$ & & $26 / 26$ & $0.99(0.53-1.88)$ & \\
\hline TA & $149 / 161$ & $\begin{array}{l}1.03(0.72- \\
1.47)\end{array}$ & $66 / 71$ & $1.04(0.71-1.51)$ & & $46 / 50$ & $1.02(0.66-1.57)$ & \\
\hline \multirow[t]{2}{*}{ AA } & $50 / 61$ & $\begin{array}{l}0.95(0.59- \\
1.52)\end{array}$ & $16 / 27$ & $0.69(0.36-1.34)$ & & $36 / 21$ & $1.91(1.08-3.37) 0.03$ & 0.120 .19 \\
\hline & & & & $P_{\text {trend }}{ }^{f}=0.60$ & & & $P_{\text {trend }}{ }^{f}=0.08$ & \\
\hline$\pi$ & $106 / 121$ & 1.0 (reference) & $40 / 46$ & $0.98(0.58-1.65)$ & & $26 / 26$ & $0.99(0.53-1.88)$ & \\
\hline TA & $149 / 161$ & 1.0 (reference) & $66 / 71$ & $0.99(0.65-1.50)$ & & $46 / 50$ & $1.10(0.64-1.63)$ & \\
\hline AA & $50 / 61$ & 1.0 (reference) & $16 / 27$ & $0.75(0.35-1.60)$ & & $36 / 21$ & $1.95(0.97-3.90) 0.06$ & 0.250 .24 \\
\hline \multicolumn{9}{|l|}{ rs2278749 } \\
\hline$C C$ & $212 / 231$ & 1.0 (reference) & 97/92 & $1.19(0.84-1.70)$ & & $66 / 58$ & $1.26(0.83-1.91)$ & \\
\hline $\mathrm{CT}$ & $86 / 87$ & $\begin{array}{l}1.19(0.83- \\
1.73)\end{array}$ & $22 / 45$ & $0.51(0.29-0.87) 0.01$ & 0.070 .15 & $33 / 32$ & $1.10(0.64-1.84)$ & \\
\hline \multirow[t]{2}{*}{$\pi$} & $7 / 19$ & $\begin{array}{l}0.40(0.16- \\
0.98)\end{array}$ & $1 / 5$ & $0.26(0.03-2.38)$ & & $5 / 6$ & $0.84(0.25-2.82)$ & \\
\hline & & & & $P_{\text {trend }}{ }^{f}=0.07$ & & & $P_{\text {trend }}{ }^{f}=0.52$ & \\
\hline $\mathrm{CC}$ & $212 / 231$ & 1.0 (reference) & $97 / 92$ & $1.19(0.84-1.70)$ & & $66 / 58$ & $1.26(0.83-1.91)$ & \\
\hline $\mathrm{CT}$ & $86 / 87$ & 1.0 (reference) & $22 / 45$ & $0.47(0.25-0.88) 0.02$ & 0.120 .18 & $33 / 32$ & $0.99(0.55-1.79)$ & \\
\hline$\pi$ & $7 / 19$ & 1.0 (reference) & $1 / 5$ & $1.33(0.10-17.78)$ & & $5 / 6$ & $2.68(0.48-15.01)$ & \\
\hline \multicolumn{9}{|l|}{ rs969485 } \\
\hline AA & $161 / 189$ & 1.0 (reference) & $51 / 74$ & $0.83(0.54-1.28)$ & & $41 / 50$ & $0.92(0.57-1.49)$ & \\
\hline$A G$ & 105/117 & $\begin{array}{l}1.05(0.74- \\
1.49)\end{array}$ & $58 / 55$ & $1.23(0.82-1.85)$ & & $50 / 35$ & $1.64(1.03-2.61) 0.04$ & 0.120 .18 \\
\hline \multirow[t]{2}{*}{ GG } & $21 / 20$ & $\begin{array}{l}1.07(0.54- \\
2.09)\end{array}$ & $8 / 8$ & $1.40(0.50-3.94)$ & & $9 / 7$ & $1.50(0.53-4.22)$ & \\
\hline & & & & $P_{\text {trend }}{ }^{f}=0.49$ & & & $P_{\text {trend }}{ }^{f}=0.05$ & \\
\hline AA & $161 / 189$ & 1.0 (reference) & $51 / 74$ & $0.83(0.54-1.28)$ & & $41 / 50$ & 0.92 (0.57 to 1.49$)$ & \\
\hline$A G$ & 105/117 & 1.0 (reference) & $58 / 55$ & $1.50(0.72-1.85)$ & & $50 / 35$ & $1.63(0.96-2.76) 0.07$ & 0.210 .23 \\
\hline GG & $21 / 20$ & 1.0 (reference) & $8 / 8$ & $1.35(0.37-4.88)$ & & $9 / 7$ & $1.60(0.38-6.74)$ & \\
\hline \multicolumn{9}{|l|}{$\begin{array}{l}\text { ARNTL2/ } \\
\text { BMAL2 }\end{array}$} \\
\hline \multicolumn{9}{|l|}{ rs2306074 } \\
\hline$\pi$ & $143 / 144$ & 1.0 (reference) & $58 / 63$ & $0.91(0.59-1.43)$ & & $51 / 53$ & $0.97(0.61-1.53)$ & \\
\hline $\mathrm{TC}$ & $134 / 163$ & $\begin{array}{l}0.84(0.60- \\
1.17)\end{array}$ & $60 / 68$ & $0.97(0.66-1.42)$ & & $50 / 40$ & $1.36(0.86-2.13)$ & \\
\hline \multirow[t]{2}{*}{$\mathrm{CC}$} & $37 / 39$ & $\begin{array}{l}1.04(0.61- \\
1.76)\end{array}$ & $6 / 21$ & $0.30(0.17-0.75) 0.01$ & 0.180 .21 & $9 / 9$ & $1.03(0.39-2.72)$ & \\
\hline & & & & $P_{\text {trend }}{ }^{\dagger}=0.06$ & & & $P_{\text {trend }}{ }^{f}=0.51$ & \\
\hline$\pi$ & $143 / 144$ & 1.0 (reference) & $58 / 63$ & $0.91(0.59-1.43)$ & & $51 / 53$ & $0.97(0.61-1.53)$ & \\
\hline $\mathrm{TC}$ & $134 / 163$ & 1.0 (reference) & $60 / 68$ & $1.06(0.68-1.64)$ & & $50 / 40$ & $1.37(0.83-2.26)$ & \\
\hline CC & $37 / 39$ & 1.0 (reference) & $6 / 21$ & $0.24(0.08-0.77) 0.02$ & 0.310 .25 & $9 / 9$ & $0.99(0.32-3.11)$ & \\
\hline \multicolumn{9}{|l|}{ CLOCK } \\
\hline \multicolumn{9}{|l|}{ rs3749474 } \\
\hline $\mathrm{CC}$ & 123/131 & 1.0 (reference) & $52 / 55$ & $0.97(0.68-1.37)$ & & $48 / 42$ & $1.08(0.73-1.59)$ & \\
\hline
\end{tabular}


Table 5 Odds ratios and $95 \%$ confidence intervals comparing odds of breast cancer associated with each polymorphism among women with less than two consecutive night shifts for 5 or more years compared to women with three consecutive night shifts or women with four or more consecutive night shifts for $\mathbf{5}$ or more years (Continued)

\begin{tabular}{|c|c|c|c|c|c|c|c|}
\hline $\mathrm{CT}$ & $148 / 152$ & $\begin{array}{l}1.04(0.74- \\
1.47)\end{array}$ & $56 / 63$ & $0.87(0.46-1.63)$ & & $47 / 45$ & $1.84(0.92-3.69)$ \\
\hline \multirow[t]{2}{*}{$\pi$} & $39 / 66$ & $\begin{array}{l}0.60(0.37- \\
0.97)\end{array}$ & $16 / 29$ & $0.24(0.05-1.18) 0.07$ & 0.560 .29 & $13 / 13$ & $3.15(0.62-16.14)$ \\
\hline & & & & $P_{\text {trend }}{ }^{f}=0.24$ & & & $P_{\text {trend }}{ }^{f}=0.63$ \\
\hline CC & 123/131 & 1.0 (reference) & $52 / 55$ & $0.97(0.68-1.37)$ & & $48 / 42$ & $1.08(0.73-1.59)$ \\
\hline CT & $148 / 152$ & 1.0 (reference) & $56 / 63$ & $0.64(0.30-1.36)$ & & $47 / 45$ & $1.44(0.63-3.31)$ \\
\hline$\pi$ & $39 / 66$ & 1.0 (reference) & $16 / 29$ & $0.30(0.05-1.77)$ & & $13 / 13$ & $2.12(0.30-15.15)$ \\
\hline \multicolumn{8}{|c|}{ CSNK1E } \\
\hline \multicolumn{8}{|c|}{ rs1534891 } \\
\hline GG & $241 / 258$ & 1.0 (reference) & $98 / 112$ & $0.93(0.66-1.309)$ & & $82 / 76$ & $1.1280 .77-1.62)$ \\
\hline GA & $62 / 77$ & $\begin{array}{l}0.83(0.57- \\
1.23)\end{array}$ & $17 / 35$ & $0.55(0.30-1.01) 0.05$ & 0.210 .23 & $25 / 25$ & $1.09(0.61-1.95)$ \\
\hline \multirow[t]{2}{*}{ AA } & $5 / 9$ & $\begin{array}{l}0.51(0.16- \\
1.60)\end{array}$ & $2 / 1$ & $2.00(0.17-23.92)$ & & $1 / 2$ & $0.39(0.03-4.68)$ \\
\hline & & & & $P_{\text {trend }}{ }^{f}=0.13$ & & & $P_{\text {trend }}{ }^{f}=0.66$ \\
\hline GG & $241 / 258$ & 1.0 (reference) & $98 / 112$ & $0.93(0.66-1.309)$ & & $82 / 76$ & $1.1280 .77-1.62)$ \\
\hline GA & $62 / 77$ & 1.0 (reference) & $17 / 35$ & $0.63(0.31-1.28)$ & & $25 / 25$ & $1.11(0.56-2.21)$ \\
\hline AA & $5 / 9$ & 1.0 (reference) & $2 / 1$ & $2.58(0.14-91.16)$ & & $1 / 2$ & $2.84(0.08-96.47)$ \\
\hline \multicolumn{8}{|c|}{ rs5757037 } \\
\hline CC & $126 / 154$ & 1.0 (reference) & $49 / 57$ & $1.10(0.68-1.76)$ & & $41 / 38$ & $1.28(0.75-2.70)$ \\
\hline$C T$ & $137 / 140$ & $\begin{array}{l}1.11(0.79- \\
1.58)\end{array}$ & $51 / 77$ & $0.67(0.45-0.99) 0.05$ & 0.130 .18 & $49 / 45$ & $1.14(0.74-1.77)$ \\
\hline \multirow[t]{2}{*}{$\pi$} & $43 / 43$ & $\begin{array}{l}1.04(0.63- \\
1.71)\end{array}$ & $14 / 16$ & $0.90(0.43-1.90)$ & & $16 / 16$ & $1.00(0.49-2.05)$ \\
\hline & & & & $P_{\text {trend }}{ }^{f}=0.57$ & & & $P_{\text {trend }}{ }^{f}=0.22$ \\
\hline CC & $126 / 154$ & 1.0 (reference) & $49 / 57$ & $1.10(0.68-1.76)$ & & $41 / 38$ & $1.28(0.75-2.70)$ \\
\hline $\mathrm{CT}$ & $137 / 140$ & 1.0 (reference) & $51 / 77$ & $0.65(0.42-1.02)$ & & $49 / 45$ & $1.08(0.66-1.75)$ \\
\hline$\pi$ & $43 / 43$ & $1.04(0.63-1.71)$ & $14 / 16$ & $0.89(0.38-2.10)$ & & $16 / 16$ & $0.97(0.42-2.23)$ \\
\hline \multicolumn{8}{|c|}{ NPAS2 } \\
\hline \multicolumn{8}{|c|}{ rs2305160 } \\
\hline GG & 136/134 & 1.0 (reference) & $52 / 57$ & $0.86(0.54-1.36)$ & & $50 / 42$ & $1.15(0.70-1.88)$ \\
\hline GA & 130/151 & $\begin{array}{l}0.84(0.59- \\
1.18)\end{array}$ & $51 / 69$ & $0.84(0.56-1.25)$ & & $43 / 41$ & $1.10(0.70-1.74)$ \\
\hline \multirow[t]{2}{*}{ AA } & $41 / 56$ & $\begin{array}{l}0.72(0.45- \\
1.18)\end{array}$ & $18 / 21$ & $0.87(0.45-1.69)$ & & $14 / 13$ & $1.22(0.56-2.64)$ \\
\hline & & & & $P_{\text {trend }}{ }^{f}=0.21$ & & & $P_{\text {trend }}{ }^{f}=0.79$ \\
\hline \multicolumn{8}{|c|}{ rs2305160 } \\
\hline GG & $136 / 134$ & 1.0 (reference) & $52 / 57$ & $0.86(0.54-1.36)$ & & $50 / 42$ & $1.15(0.70-1.88)$ \\
\hline GA & 130/151 & 1.0 (reference) & $51 / 69$ & $0.95(0.61-1.50)$ & & $43 / 41$ & $1.19(0.72-1.96)$ \\
\hline AA & $41 / 56$ & 1.0 (reference) & $18 / 21$ & $1.10(0.50-2.40)$ & & $14 / 13$ & $1.43(0.58-3.52)$ \\
\hline \multicolumn{8}{|c|}{ rs17024926 } \\
\hline$\pi$ & 110/133 & 1.0 (reference) & $60 / 66$ & $1.22(0.78-1.92)$ & & $36 / 45$ & $1.03(0.61-1.75)$ \\
\hline TC & $148 / 178$ & $\begin{array}{l}1.08(0.76- \\
1.52)\end{array}$ & $55 / 64$ & $0.98(0.66-1.47)$ & & $54 / 46$ & $1.23(0.80-1.89)$ \\
\hline \multirow[t]{2}{*}{ CC } & $47 / 38$ & $\begin{array}{l}1.51(0.90- \\
2.52)\end{array}$ & $6 / 21$ & $\begin{array}{l}0.33(0.13-0.84) \\
0.020\end{array}$ & 0.240 .23 & $17 / 10$ & $1.89(0.85-4.23)$ \\
\hline & & & & $P_{\text {trend }}{ }^{f}=0.28$ & & & $P_{\text {trend }}{ }^{f}=0.05$ \\
\hline$\pi$ & 110/133 & 1.0 (reference) & $60 / 66$ & $1.22(0.78-1.92)$ & & $36 / 45$ & $1.03(0.61-1.75)$ \\
\hline TC & $148 / 178$ & 1.0 (reference) & $55 / 64$ & $1.01(0.65-1.57)$ & & $54 / 46$ & $1.28(0.81-2.04)$ \\
\hline CC & $47 / 38$ & 1.0 (reference) & $6 / 21$ & $\begin{array}{l}0.24(0.09-0.67) \\
0.006\end{array}$ & 0.190 .22 & $17 / 10$ & $1.49(0.56-3.95)$ \\
\hline
\end{tabular}


Table 5 Odds ratios and $95 \%$ confidence intervals comparing odds of breast cancer associated with each polymorphism among women with less than two consecutive night shifts for 5 or more years compared to women with three consecutive night shifts or women with four or more consecutive night shifts for $\mathbf{5}$ or more years (Continued)

\begin{tabular}{|c|c|c|c|c|c|c|c|c|}
\hline \multicolumn{9}{|c|}{ rs11695472 } \\
\hline AA & $173 / 187$ & 1.0 (reference) & $68 / 87$ & $0.87(0.59-1.29)$ & & $63 / 48$ & $1.37(0.88-2.14)$ & \\
\hline$A C$ & $117 / 143$ & $\begin{array}{l}0.90(0.65- \\
1.25)\end{array}$ & $38 / 54$ & $0.74(0.47-1.16)$ & & $36 / 44$ & $0.87(0.54-1.39)$ & \\
\hline \multirow[t]{2}{*}{ CC } & $22 / 21$ & $\begin{array}{l}1.24(0.64- \\
2.39)\end{array}$ & $16 / 7$ & $2.69(1.08-6.73) 0.03$ & 0.280 .25 & $9 / 8$ & $1.32(0.48-3.65)$ & \\
\hline & & & & $P_{\text {trend }}{ }^{f}=0.93$ & & & $P_{\text {trend }}{ }^{f}=0.79$ & \\
\hline AA & 173/187 & 1.0 (reference) & $68 / 87$ & $0.87(0.59-1.29)$ & & $63 / 48$ & $1.37(0.88-2.14)$ & \\
\hline$A C$ & $117 / 143$ & 1.0 (reference) & $38 / 54$ & $0.83(0.50-1.39)$ & & $36 / 44$ & $0.93(0.55-1.55)$ & \\
\hline CC & $22 / 21$ & 1.0 (reference) & $16 / 7$ & $1.91(0.59-6.22)$ & & $9 / 8$ & $0.83(0.22-3.14)$ & \\
\hline \multicolumn{9}{|c|}{ PER3 } \\
\hline \multicolumn{9}{|c|}{ rs1012477 } \\
\hline GG & 203/237 & 1.0 (reference) & $88 / 97$ & $1.12(0.77-1.58)$ & & $66 / 73$ & $1.02(0.69-1.51)$ & \\
\hline GC & $95 / 100$ & $\begin{array}{l}1.10(0.78- \\
1.56)\end{array}$ & $22 / 51$ & $\begin{array}{l}0.42(0.24-0.72) \\
0.002\end{array}$ & 0.020 .08 & $35 / 24$ & $1.56(0.91-2.68)$ & \\
\hline \multirow[t]{2}{*}{ CC } & $9 / 7$ & $\begin{array}{l}1.69(0.58- \\
4.93)\end{array}$ & $3 / 1$ & $3.42(0.35-33.40)$ & & $3 / 2$ & $1.90(0.31-11.58)$ & \\
\hline & & & & $P_{\text {trend }}{ }^{f}=0.15$ & & & $P_{\text {trend }}{ }^{f}=0.08$ & \\
\hline GG & 203/237 & 1.0 (reference) & $88 / 97$ & $1.12(0.77-1.58)$ & & $66 / 73$ & $1.02(0.69-1.51)$ & \\
\hline GC & $95 / 100$ & 1.0 (reference) & $22 / 51$ & $\begin{array}{l}0.38(0.18-0.66) \\
0.001\end{array}$ & 0.010 .06 & $35 / 24$ & $1.46(0.79-2.72)$ & \\
\hline CC & $9 / 7$ & 1.0 (reference) & $3 / 1$ & $3.00(0.12-77.00)$ & & $3 / 2$ & $1.30(0.06-28.93)$ & \\
\hline \multicolumn{9}{|c|}{$R O R-b$} \\
\hline \multicolumn{9}{|c|}{ rs3903529 } \\
\hline$\pi$ & $162 / 183$ & 1.0 (reference) & $64 / 70$ & $1.09(0.71-1.66)$ & & $51 / 47$ & $1.20(0.75-1.93)$ & \\
\hline TA & 123/131 & $\begin{array}{l}1.08(0.77- \\
1.51)\end{array}$ & $41 / 62$ & $0.75(0.49-1.15)$ & & $40 / 47$ & $0.92(0.59-1.46)$ & \\
\hline \multirow[t]{2}{*}{ AA } & $22 / 27$ & $\begin{array}{l}0.98(0.53- \\
1.82)\end{array}$ & $13 / 18$ & $0.76(0.36-1.59)$ & & $14 / 5$ & $\begin{array}{l}2.77(0.98-7.86) \\
0.056\end{array}$ & 0.380 .27 \\
\hline & & & & $P_{\text {trend }}{ }^{f}=0.26$ & & & $P_{\text {trend }^{f}}{ }^{f}=0.21$ & \\
\hline$\Pi$ & $162 / 183$ & 1.0 (reference) & $64 / 70$ & $1.09(0.71-1.66)$ & & $51 / 47$ & $1.20(0.75-1.93)$ & \\
\hline TA & $123 / 131$ & 1.0 (reference) & $41 / 62$ & $0.72(0.44-1.16)$ & & $40 / 47$ & $0.94(0.57-1.55)$ & \\
\hline AA & $22 / 27$ & 1.0 (reference) & $13 / 18$ & $0.78(0.30-2.05)$ & & $14 / 5$ & $3.07(0.84-11.18)$ & \\
\hline \multicolumn{9}{|c|}{ rs7022435 } \\
\hline GG & 178/194 & 1.0 (reference) & $72 / 85$ & $0.97(0.65-1.43)$ & & $55 / 56$ & $1.05(0.67-1.64)$ & \\
\hline GA & $115 / 128$ & $\begin{array}{l}1.00(0.72- \\
1.40)\end{array}$ & $44 / 49$ & $1.02(0.65-1.60)$ & & $41 / 32$ & $1.38(0.84-2.26)$ & \\
\hline \multirow[t]{2}{*}{ AA } & $15 / 19$ & $\begin{array}{l}0.93(0.45- \\
1.90)\end{array}$ & $3 / 12$ & $0.25(0.07-0.89) 0.03$ & 0.410 .27 & $11 / 8$ & $1.34(0.52-3.41)$ & \\
\hline & & & & $P_{\text {trend }}{ }^{f}=0.25$ & & & $P_{\text {trend }}{ }^{f}=0.15$ & \\
\hline GG & 178/194 & 1.0 (reference) & $72 / 85$ & $0.97(0.65-1.43)$ & & $55 / 56$ & $1.05(0.67-1.64)$ & \\
\hline GA & $115 / 128$ & 1.0 (reference) & $44 / 49$ & $1.03(0.62-1.70)$ & & $41 / 32$ & $1.34(0.78-2.92)$ & \\
\hline AA & $15 / 19$ & 1.0 (reference) & $3 / 12$ & $0.29(0.06-1.33)$ & & $11 / 8$ & $2.18(0.55-8.61)$ & \\
\hline \multicolumn{9}{|c|}{ rs3750420 } \\
\hline CC & $136 / 166$ & 1.0 (reference) & $52 / 66$ & $0.99(0.63-1.55)$ & & $41 / 47$ & $1.02(0.61-1.68)$ & \\
\hline$C T$ & $136 / 143$ & $\begin{array}{l}1.20(0.86- \\
1.68)\end{array}$ & $60 / 58$ & $1.24(0.83-1.86)$ & & $48 / 35$ & $1.60(1.00-2.56) 0.05$ & 0.150 .20 \\
\hline \multirow[t]{2}{*}{$\pi$} & $28 / 31$ & $\begin{array}{l}1.18(0.67- \\
2.08)\end{array}$ & $6 / 18$ & $0.37(0.14-0.96) 0.04$ & 0.310 .25 & $17 / 12$ & $1.52(0.71-3.28)$ & \\
\hline & & & & $P_{\text {trend }}{ }^{f}=0.82$ & & & $P_{\text {trend }}{ }^{f}=\mathbf{0 . 0 3}$ & \\
\hline CC & $136 / 166$ & 1.0 (reference) & $52 / 66$ & $0.99(0.63-1.55)$ & & $41 / 47$ & $1.02(0.61-1.68)$ & \\
\hline$C T$ & $136 / 143$ & 1.0 (reference) & $60 / 58$ & $1.13(0.72-1.77)$ & & $48 / 35$ & $1.47(0.88-2.45)$ & \\
\hline$\pi$ & $28 / 31$ & 1.0 (reference) & $6 / 18$ & $0.33(0.11-1.00) 0.05$ & 0.390 .27 & $17 / 12$ & $1.39(0.54-3.60)$ & \\
\hline
\end{tabular}


Table 5 Odds ratios and $95 \%$ confidence intervals comparing odds of breast cancer associated with each polymorphism among women with less than two consecutive night shifts for 5 or more years compared to women with three consecutive night shifts or women with four or more consecutive night shifts for $\mathbf{5}$ or more years (Continued)

\begin{tabular}{|c|c|c|c|c|c|c|c|c|}
\hline \multicolumn{9}{|c|}{ MTNR1A } \\
\hline \multicolumn{9}{|c|}{ rs13113549 } \\
\hline$\pi$ & 113/109 & 1.0 (reference) & $48 / 54$ & $0.94(0.57-1.53)$ & & $35 / 33$ & $1.09(0.62-1.93)$ & \\
\hline $\mathrm{TC}$ & $142 / 155$ & $\begin{array}{l}0.94(0.66- \\
1.35)\end{array}$ & $59 / 60$ & $1.08(0.72-1.61)$ & & $52 / 49$ & $1.08(0.71-1.65)$ & \\
\hline \multirow[t]{2}{*}{$\mathrm{CC}$} & $55 / 75$ & $\begin{array}{l}0.75(0.48- \\
1.18)\end{array}$ & $16 / 34$ & $0.47(0.25-0.91) 0.02$ & 0.150 .20 & $22 / 18$ & $1.24(0.65-2.39)$ & \\
\hline & & & & $P_{\text {trend }}^{f}=0.08$ & & & $P_{\text {trend }}{ }^{f}=0.71$ & \\
\hline$\pi$ & $113 / 109$ & 1.0 (reference) & $48 / 54$ & $0.94(0.57-1.53)$ & & $35 / 33$ & $1.09(0.62-1.93)$ & \\
\hline $\mathrm{TC}$ & $142 / 155$ & 1.0 (reference) & $59 / 60$ & $1.03(0.66-1.61)$ & & $52 / 49$ & $1.09(0.68-1.75)$ & \\
\hline $\mathrm{CC}$ & $55 / 75$ & 1.0 (reference) & $16 / 34$ & $0.57(0.27-0.19)$ & & $22 / 18$ & $1.28(0.61-2.68)$ & \\
\hline \multicolumn{9}{|c|}{ MTNR1B } \\
\hline \multicolumn{9}{|c|}{ rs10830963 } \\
\hline $\mathrm{CC}$ & $176 / 195$ & 1.0 (reference) & $63 / 84$ & $0.83(0.58-1.23)$ & & $51 / 50$ & $1.01(0.64-1.61)$ & \\
\hline CG & $105 / 123$ & $\begin{array}{l}0.86(0.61- \\
1.21)\end{array}$ & $46 / 51$ & $1.06(0.69-1.64)$ & & $46 / 45$ & $1.13(0.73-1.75)$ & \\
\hline \multirow[t]{2}{*}{ GG } & $21 / 21$ & $\begin{array}{l}1.05(0.55- \\
2.02)\end{array}$ & $11 / 7$ & $1.59(0.59-4.25)$ & & $9 / 1$ & $\begin{array}{l}9.12(1.14-73.09) \\
0.04\end{array}$ & 0.590 .29 \\
\hline & & & & $P_{\text {trend }}{ }^{f}=0.76$ & & & $P_{\text {trend }}{ }^{f}=0.10$ & \\
\hline $\mathrm{CC}$ & $176 / 195$ & 1.0 (reference) & $63 / 84$ & $0.83(0.58-1.23)$ & & $51 / 50$ & $1.01(0.64-1.61)$ & \\
\hline CG & $105 / 123$ & 1.0 (reference) & $46 / 51$ & $1.17(0.71-1.93)$ & & $46 / 45$ & $1.26(0.76-2.08)$ & \\
\hline GG & $21 / 21$ & 1.0 (reference) & $11 / 7$ & $1.44(0.43-4.86)$ & & $9 / 1$ & $9.45(0.97-91.82)$ & \\
\hline
\end{tabular}

${ }^{a}$ Number of cases and controls that were successfully genotyped. ${ }^{b}$ OR derived from unconditional logistic regression, including an interaction term between the night shift variable and the SNP genotypes, and adjusted for age at diagnosis (cases) or interview (controls), selection period, alcohol use, hormone replacement therapy for the past 2 years, number of children and history of breast cancer in first-degree family members (mother or sister). ${ }^{c} P$ interaction was obtained from the interaction term between the SNP genotypes and the night shift variable using frequency of the common genotype in the 0 to 2 night shift group as reference. Only significant or near-significant values are shown. ${ }^{\mathrm{d}}$ The false-positive report probability (FPRP) was calculated as described by Wacholder et al. [42]. FPRP $<0.2$ was considered noteworthy. ${ }^{\mathrm{e} T h e}$ Bayesian false discovery probability (BFDP) was calculated as described by Wakefield et al. [41]. BFDP < 0.8 was considered noteworthy. For more details on FPRP and BFDP, see the Methods section. ${ }^{f} P_{\text {trend }}$ values for Cochran-Armitage trend test were calculated from $2 \times C$ tables using frequency of genotypes in cases and controls from 0 to 2 night shift group as reference.

breast cancer risk (data not shown). If these SNPs, or others in linkage disequilibrium with them, may represent susceptibility variants for breast cancer, it is possible they do so by altering the gene expression of the ROR- $b$ gene. The ROR proteins are involved in regulation of circadian rhythms by activating transcription of the core circadian $B M A L 1$ gene [56].

Two polymorphisms in the CRY2 gene (rs11038689 and rs1401417) had protective effects in women with four or more night shifts. The biological functions of these SNPs are not known. These polymorphisms have been found to be associated with postmenopausal breast cancer risk, with significant effect modification by menopausal status where this association was evident only in women with ER- and/or PR-negative breast tumors but not ER- and/or PR-positive tumors [57]. This may indicate that these polymorphisms may influence breast cancer risk through estrogen and progesterone signaling pathways. ERs and PRs are important for reproductive performance in women. Kovanen et al. [46] showed that women with BMAL1 rs2278749 TT genotypes had more pregnancies and miscarriages, whereas NPAS2 rs11673746 T carriers had fewer miscarriages, indicating a possible role of these polymorphisms affecting age at first birth and also number of children, both of which are established risk factors for breast cancer [58].

In the genes controlling melatonin biosynthesis (AANAT) or melatonin receptors (MTNR1A and MTNR1B), several SNPs were associated with increased risk of breast cancer. In the main effects analysis, the variant genotypes of both of the AANAT polymorphisms were associated with increased breast cancer risk. However, the risk associated with the rs3760138 GG genotype was $40 \%$ higher in the most exposed group with four or more night shifts (1.82 vs 1.42). These two SNPs are located in the regulatory regions of the $A A N A T$ gene which could potentially affect the expression of the $A A N A T$ gene. The $A A N A T$ gene product is an acetyltransferase that regulates melatonin biosynthesis during night and day periods by pineal gland. Polymorphisms in this gene have also been associated with sleep disorders, but there are no data on the association between these polymorphisms and breast cancer risk. The levels of melatonin may also be affected by variants in the genes that encode melatonin receptors $1 \mathrm{~A}$ and $1 \mathrm{~B}$. The MTNR1B 
rs10830963 was associated with increased risk in the highlevel exposed group of women carrying two variant alleles, without being noteworthy by FPRP and BFDP. The variant alleles of MTNR1A rs113113549 polymorphisms were associated with decreased risk of breast cancer in the women with three consecutive night shifts. Night shift workers have been shown to have substantially reduced melatonin levels during night work and daytime sleep, and levels remain low even when a night shift worker sleeps at night [22].

\section{Strengths and limitations of the study}

Our results indicate that some SNPs of the core circadian or melatonin signaling pathways, in combination with night work, may affect breast cancer risk. These findings are consistent with the results of recent studies, including our previous study of the same nurses, suggesting a higher risk with longer duration of intense night shift work per week $[7,9,10]$. The reported associations are biologically plausible, as the polymorphisms in the genes investigated could influence gene expression, protein function and protein-protein interactions, all of which may affect several known risk factors for breast cancer. However, this is a medium-sized, exploratory study highlighting the need for investigation of possible combinatory effects of genetic polymorphisms and night shift work in breast cancer. The results should be interpreted with caution, and larger studies are needed to confirm the associations found here.

The study may have a number of limitations. A larger reference group without any night shift work is needed. Our decision to use women with maximum of two consecutive night shifts as the reference group was made to ensure a sufficient number of subjects for each genotype. Furthermore, the metrics of exposure to night work used in our study are different from those used in other studies; therefore, the results may not be comparable. We have selected a limited number of polymorphisms, and, to cover all SNPs in the core circadian and melatonin signaling pathways, a much larger study (that is, GWAS) is warranted. The selection of genes and polymorphisms differs from other studies as well and may have affected the outcomes. Finally, although we set very low prior probabilities for multiple testing, it is still possible that some associations may not be true positive or negative findings.

\section{Conclusion}

We have found a number of polymorphisms in a number of core circadian or melatonin biosynthesis and/or bioavailability pathway genes that could be associated with breast cancer risk in shift workers. The associations seem biologically plausible because many of the polymorphisms may alter gene expression, mRNA stability or protein function. Future studies investigating the genetic and epigenetic functionality of these SNPs, as well as epigenetic changes in the shift workers, are warranted to understand exact biological mechanisms.

\section{Additional material}

\section{Additional file 1: Legend to Supplementary Figure 1: The flow-chart} showing study design.

Additional file 2: Supplementary Tables S1-Table S2. Supplementary Table S1: Full name of the circadian genes and their protein functions. Supplementary Table S2: The circadian genes, SNPs, base change, MAF/ GMAF, Hardy-Weinberg values and genotyping rate

\section{Abbreviations}

AANAT: Arylalkylamine N-acetyltransferase; ARNTL: Aryl hydrocarbon receptor nuclear translocator-like; CGEMS: Cancer genetic marker of susceptibility; CIGENE: Centre for Integrative Genetics; CLOCK: Circadian locomotor output cycle kaput; CRY: Cryptochrome; CSNK1E: Casein kinase 1; ER: Estrogen receptor; GWAS: Genome-wide association study; H-W: Hardy-Weinberg; LAN: Light at night; MAF: Minor allele frequency; MTNR: Melatonin receptor; NPAS2: Neuronal PAS domain protein 2; PER: Period; ROR: RAR-related orphan receptor; SNP: Single-nucleotide polymorphism

\section{Competing interests}

The authors declare that they have no competing interests.

\section{Authors' contributions}

$\mathrm{SZ}, \mathrm{AH}$ and $\mathrm{KHA}$ were involved in designing and conducting the genetic studies, genotyping and data analysis and wrote the first and revised drafts of the manuscript. JASL, HK and KK were responsible for the design, coordination and execution of the case-control study and were also involved in revising the manuscript. All authors read and approved the final manuscript.

\section{Acknowledgements}

The technical assistance of Tove Andreassen is greatly acknowledged. This project was supported by the Research Council of Norway and the Norwegian Cancer Society.

\section{Authors' details}

${ }^{1}$ Department of Chemical and Biological Work Environment, National Institute of Occupational Health, PB 8149 Department, N-0033 Oslo, Norway. ${ }^{2}$ Department of Occupational Medicine and Epidemiology, National Institute of Occupational Health, Oslo, Norway. ${ }^{3}$ Cancer Registry of Norway, Institute of Population-based Cancer Research, Oslo, Norway.

Received: 24 October 2012 Revised: 21 April 2013

Accepted: 3 July 2013 Published: 3 July 2013

\section{References}

1. Downs-Holmes C, Silverman P: Breast cancer: overview \& updates. Nurse Pract 2011, 36:20-27.

2. Lie JAS, Andersen A, Kjærheim K: Cancer risk among 43000 Norwegian nurses. Scand J Work Environ Health 2007, 33:66-73.

3. Poli A, Marangoni F, Visioli F: Alcohol consumption and breast cancer risk. JAMA 2012, 307:666.

4. Ghoussaini M, Fletcher O, Michailidou K, Turnbull C, Schmidt MK, Dicks E, Dennis J, Wang Q, Humphreys MK, Luccarini C, Baynes C, Conroy D, Maranian M, Ahmed S, Driver K, Johnson N, Orr N, dos Santos Silva I, Waisfisz Q, Meijers-Heijboer H, Uitterlinden AG, Rivadeneira F, Netherlands Collaborative Group on Hereditary Breast and Ovarian Cancer (HEBON), Hall P, Czene K, Irwanto A, Liu J, Nevanlinna H, Aittomäki K, Blomqvist C, Meindl $A$, et al: Genome-wide association analysis identifies three new breast cancer susceptibility loci. Nat Genet 2012, 44:312-318.

5. Turnbull C, Seal S, Renwick A, Warren-Perry M, Hughes D, Elliott A, Pernet D, Peock S, Adlard JW, Barwell J, Berg J, Brady AF, Brewer C, Brice G, 
Chapman C, Cook J, Davidson R, Donaldson A, Douglas F, Greenhalgh L, Henderson A, Izatt L, Kumar A, Lalloo F, Miedzybrodzka Z, Morrison PJ, Paterson J, Porteous M, Rogers MT, Shanley S, Walker L, Breast Cancer Susceptibility Collaboration (UK), Ahmed M, Eccles D, Evans DG, Donnelly P, Easton DF, Stratton MR, Rahman N: Gene-gene interactions in breast cancer susceptibility. Hum Mol Genet 2012, 21:958-962.

6. Hansen J, Stevens RG: Case-control study of shift-work and breast cancer risk in Danish nurses: impact of shift systems. Eur J Cancer 2012, 48:1722-1729.

7. Hansen J, Lassen CF: Nested case-control study of night shift work and breast cancer risk among women in the Danish military. Occup Environ Med 2012, 69:551-556.

8. Lie JA, Roessink J, Kjærheim K: Breast cancer and night work among Norwegian nurses. Cancer Causes Control 2006, 17:39-44.

9. Lie JA, Kjuus H, Zienolddiny S, Haugen A, Stevens RG, Kjærheim K: Night work and breast cancer risk among Norwegian nurses: assessment by different exposure metrics. Am J Epidemiol 2011, 173:1272-1279.

10. Menegaux F, Truong T, Anger A, Cordina-Duverger E, Lamkarkach F, Arveux P, Kerbrat P, Févotte J, Guénel P: Night work and breast cancer: a population-based case-control study in France (the CECILE study). Int J Cancer 2013, 132:924-931.

11. Pukkala E, Helminen M, Haldorsen T, Hammar N, Kojo K, Linnersjö A Rafnsson V, Tulinius H, Tveten U, Auvinen A: Cancer incidence among Nordic airline cabin crew. Int J Cancer 2012, 131:2886-2897.

12. Schernhammer ES, Kroenke CH, Laden F, Hankinson SE: Night work and risk of breast cancer. Epidemiology 2006, 17:108-111.

13. Rushton L, Hutchings SJ, Fortunato L, Young C, Evans GS, Brown T, Bevan R, Slack R, Holmes P, Bagga S, Cherrie JW, Van Tongeren M: Occupational cancer burden in Great Britain. Br J Cancer 2012, 107(Suppl 1):S3-S7.

14. Schwartzbaum J, Ahlbom A, Feychting M: Cohort study of cancer risk among male and female shift workers. Scand I Work Environ Health 2007, 33:336-343.

15. Pesch B, Harth V, Rabstein S, Baisch C, Schiffermann M, Pallapies D, Bonberg N, Heinze E, Spickenheuer A, Justenhoven C, Brauch H, Hamann U, Ko Y, Straif K, Brüning T: Night work and breast cancer: results from the German GENICA study. Scand I Work Environ Health 2010, 36:134-141.

16. O'Leary ES, Schoenfeld ER, Stevens RG, Kabat GC, Henderson K, Grimson R, Gammon MD, Leske MC: Shift work, light at night, and breast cancer on Long Island, New York. Am J Epidemiol 2006, 164:358-366.

17. IARC Working Group on the Evaluation of Carcinogenic Risks to Humans: Painting, firefighting, and shiftwork. IARC Monogr Eval Carcinog Risks Hum 2010, 98:9-764.

18. Straif K, Baan R, Grosse Y, Secretan B, El Ghissassi F, Bouvard V, Altieri A, Benbrahim-Tallaa L, Cogliano V: Carcinogenicity of shift-work, painting, and fire-fighting. Lancet Oncol 2007, 8:1065-1066.

19. Stevens RG: Testing the light-at-night (LAN) theory for breast cancer causation. Chronobiol Int 2011, 28:653-656.

20. Blask DE, Hill SM, Dauchy RT, Xiang S, Yuan L, Duplessis T, Mao L, Dauchy E, Sauer LA: Circadian regulation of molecular, dietary, and metabolic signaling mechanisms of human breast cancer growth by the nocturnal melatonin signal and the consequences of its disruption by light at night. J Pineal Res 2011, 51:259-269.

21. Stevens RG: Circadian disruption and breast cancer: from melatonin to clock genes. Epidemiology 2005, 16:254-258.

22. Davis S, Mirick DK, Chen C, Stanczyk FZ: Night shift work and hormone levels in women. Cancer Epidemiol Biomarkers Prev 2012, 21:609-618.

23. Dumont M, Lanctôt V, Cadieux-Viau R, Paquet J: Melatonin production and light exposure of rotating night workers. Chronobiol Int 2012, 29:203-210.

24. Slominski RM, Reiter RJ, Schlabritz-Loutsevitch N, Ostrom RS, Slominski AT: Melatonin membrane receptors in peripheral tissues: distribution and functions. Mol Cell Endocrinol 2012, 351:152-166.

25. Hill SM, Spriggs LL, Simon MA, Muraoka H, Blask DE: The growth inhibitory action of melatonin on human breast cancer cells is linked to the estrogen response system. Cancer Lett 1992, 64:249-256.

26. Hill SM, Collins A, Kiefer TL: The modulation of oestrogen receptor-a activity by melatonin in MCF-7 human breast cancer cells. Eur J Cancer 2000, 36(Suppl 4):117-118.

27. Sánchez-Hidalgo M, Lee M, de la Lastra CA, Guerrero JM, Packham G: Melatonin inhibits cell proliferation and induces caspase activation and apoptosis in human malignant lymphoid cell lines. J Pineal Res 2012, 53:366-373.
28. Tamura H, Takasaki A, Taketani T, Tanabe M, Kizuka F, Lee L, Tamura I, Maekawa R, Asada H, Yamagata Y, Sugino N: Melatonin as a free radical scavenger in the ovarian follicle. Endocr J 2013, 60:1-13.

29. Morceli G, Honorio-França AC, Fagundes DL, Calderon IM, França EL: Antioxidant effect of melatonin on the functional activity of colostral phagocytes in diabetic women. PLoS One 2013, 8:e56915.

30. Haus EL, Smolensky MH: Shift work and cancer risk: potential mechanistic roles of circadian disruption, light at night, and sleep deprivation. Sleep Med Rev 2013, 17:273-284.

31. Pinheiro SP, Schernhammer ES, Tworoger SS, Michels KB: A prospective study on habitual duration of sleep and incidence of breast cancer in a large cohort of women. Cancer Res 2006, 66:5521-5525.

32. Girschik J, Heyworth J, Fritschi L: Self-reported sleep duration, sleep quality, and breast cancer risk in a population-based case-control study. Am J Epidemiol 2013, 177:316-327.

33. Griffett K, Burris TP: The mammalian clock and chronopharmacology. Bioorg Med Chem Lett 2013, 23:1929-1934.

34. Panda S, Sato TK, Castrucci AM, Rollag MD, DeGrip WJ, Hogenesch JB, Provencio I, Kay SA: Melanopsin (Opn4) requirement for normal lightinduced circadian phase shifting. Science 2002, 298:2213-2216.

35. Zmrzljak UP, Rozman D: Circadian regulation of the hepatic endobiotic and xenobiotic detoxification pathways: the time matters. Chem Res Toxicol 2012, 25:811-824.

36. Gery S, Koeffler HP: The role of circadian regulation in cancer. Cold Spring Harb Symp Quant Biol 2007, 72:459-464.

37. Hua H, Wang Y, Wan C, Liu Y, Zhu B, Yang C, Wang X, Wang Z, CornelissenGuillaume G, Halberg F: Circadian gene mPer2 overexpression induces cancer cell apoptosis. Cancer Sci 2006, 97:589-596.

38. Gu X, Xing L, Shi G, Liu Z, Wang X, Qu Z, Wu X, Dong Z, Gao X, Liu G, Yang $L, X U$ Y: The circadian mutation PER2S662G is linked to cell cycle progression and tumorigenesis. Cell Death Differ 2012, 19:397-405.

39. Dai H, Zhang L, Cao M, Song F, Zheng H, Zhu X, Wei Q, Zhang W, Chen K: The role of polymorphisms in circadian pathway genes in breast tumorigenesis. Breast Cancer Res Treat 2011, 127:531-540.

40. Larsen IK, Småstuen M, Johannesen TB, Langmark F, Parkin DM, Bray F, Møller B: Data quality at the Cancer Registry of Norway: an overview of comparability, completeness, validity and timeliness. Eur J Cancer 2009, 45:1218-1231.

41. Wakefield J: A Bayesian measure of the probability of false discovery in genetic epidemiology studies. Am J Hum Genet 2007, 81:208-227.

42. Wacholder S, Chanock S, Garcia-Closas M, El Ghormli L, Rothman N: Assessing the probability that a positive report is false: an approach for molecular epidemiology studies. J Natl Cancer Inst 2004, 96:434-442.

43. Wacholder S, Struewing JP, Hartge P, Greene MH, Tucker MA: Breast cancer risks for BRCA1/2 carriers. Science 2004, 306:2187-2191.

44. Hung RJ, Brennan P, Canzian F, Szeszenia-Dabrowska N, Zaridze D, Lissowska J, Rudnai P, Fabianova E, Mates D, Foretova L, Janout V, Bencko V, Chabrier A, Borel S, Hall J, Boffetta P: Large-scale investigation of base excision repair genetic polymorphisms and lung cancer risk in a multicenter study. J Natl Cancer Inst 2005, 97:567-576.

45. Kovanen L, Saarikoski ST, Haukka J, Pirkola S, Aromaa A, Lönnqvist J, Partonen T: Circadian clock gene polymorphisms in alcohol use disorders and alcohol consumption. Alcohol Alcohol 2010, 45:303-311.

46. Kovanen L, Saarikoski ST, Aromaa A, Lönnqvist J, Partonen T: ARNTL (BMAL1) and NPAS2 gene variants contribute to fertility and seasonality. PLOS One 2010, 5:e10007.

47. Zhu Y, Stevens RG, Hoffman AE, Fitzgerald LM, Kwon EM, Ostrander EA, Davis S, Zheng T, Stanford JL: Testing the circadian gene hypothesis in prostate cancer: a population-based case-control study. Cancer Res 2009, 69:9315-9322

48. Hoffman AE, Zheng T, Ba Y, Zhu Y: The circadian gene NPAS2, a putative tumor suppressor, is involved in DNA damage response. Mol Cancer Res 2008, 6:1461-1468.

49. Hoffman $A E$, Zheng $T, B a Y$, Stevens $R G, Y i C H$, Leaderer $D$, Zhu $Y$ : Phenotypic effects of the circadian gene cryptochrome 2 on cancerrelated pathways. BMC Cancer 2010, 10:110.

50. Hoffman AE, Yi CH, Zheng T, Stevens RG, Leaderer D, Zhang Y, Holford TR, Hansen J, Paulson J, Zhu Y: CLOCK in breast tumorigenesis: genetic, epigenetic, and transcriptional profiling analyses. Cancer Res 2010, 70:1459-1468. 
51. Zhou F, He X, Liu H, Zhu Y, Jin T, Chen C, Qu F, Li Y, Bao G, Chen Z, Xing J: Functional polymorphisms of circadian positive feedback regulation genes and clinical outcome of Chinese patients with resected colorectal cancer. Cancer 2012, 118:937-946.

52. Reszka E, Peplonska B, Wieczorek E, Sobala W, Bukowska A, Gromadzinska J, Lie JA, Kjuus H, Wasowicz W: Circadian gene expression in peripheral blood leukocytes of rotating night shift nurses. Scand J Work Environ Health 2013, 39:187-194.

53. Reszka E, Peplonska B, Wieczorek E, Sobala W, Bukowska A, Gromadzinska J, Lie JA, Kjuus H, Wasowicz W: Rotating night shift work and polymorphism of genes important for the regulation of circadian rhythm. Scand J Work Environ Health 2013, 39:178-186.

54. Monsees GM, Kraft P, Hankinson SE, Hunter DJ, Schernhammer ES: Circadian genes and breast cancer susceptibility in rotating shift workers. Int J Cancer 2012, 131:2547-2552.

55. McGrath CL, Glatt SJ, Sklar P, Le-Niculescu H, Kuczenski R, Doyle AE, Biederman J, Mick E, Faraone SV, Niculescu AB, Tsuang MT: Evidence for genetic association of RORB with bipolar disorder. BMC Psychiatry 2009, 9:70.

56. Akashi M, Takumi T: The orphan nuclear receptor RORa regulates circadian transcription of the mammalian core-clock Bmal1. Nat Struct Mol Biol 2005, 12:441-448.

57. Hoffman $A E$, Zheng $T$, Yi CH, Stevens RG, Ba Y, Zhang $Y$, Leaderer $D$, Holford T, Hansen J, Zhu Y: The core circadian gene Cryptochrome 2 influences breast cancer risk, possibly by mediating hormone signaling. Cancer Prev Res (Phila) 2010, 3:539-548.

58. Boden MJ, Kennaway DJ: Circadian rhythms and reproduction Reproduction 2006, 132:379-392.

doi:10.1186/bcr3445

Cite this article as: Zienolddiny et al: Analysis of polymorphisms in the circadian-related genes and breast cancer risk in Norwegian nurses working night shifts. Breast Cancer Research 2013 15:R53.

\section{Submit your next manuscript to BioMed Central and take full advantage of:}

- Convenient online submission

- Thorough peer review

- No space constraints or color figure charges

- Immediate publication on acceptance

- Inclusion in PubMed, CAS, Scopus and Google Scholar

- Research which is freely available for redistribution

Submit your manuscript at www.biomedcentral.com/submit
Biomed Central 